\title{
The Antifungal Pipeline: Fosmanogepix, Ibrexafungerp, Olorofim, Opelconazole, and Rezafungin
}

\author{
Martin Hoenigl ${ }^{1,2,3}$ (1) $\cdot$ Rosanne Sprute ${ }^{4,5,6} \cdot$ Matthias Egger $^{1} \cdot$ Amir Arastehfar $^{7}$. Oliver A. Cornely ${ }^{4,5,6,8}$. \\ Robert Krause $^{1} \cdot$ Cornelia Lass-Flörl $^{9} \cdot$ Juergen Prattes ${ }^{1} \cdot$ Andrej Spec $^{10} \cdot$ George R. Thompson III $^{11}$. \\ Nathan Wiederhold ${ }^{12}$. Jeffrey D. Jenks ${ }^{2,3,13}$
}

Accepted: 9 September 2021 / Published online: 9 October 2021

(c) The Author(s) 2021

\begin{abstract}
The epidemiology of invasive fungal infections is changing, with new populations at risk and the emergence of resistance caused by the selective pressure from increased usage of antifungal agents in prophylaxis, empiric therapy, and agriculture. Limited antifungal therapeutic options are further challenged by drug-drug interactions, toxicity, and constraints in administration routes. Despite the need for more antifungal drug options, no new classes of antifungal drugs have become available over the last 2 decades, and only one single new agent from a known antifungal class has been approved in the last decade. Nevertheless, there is hope on the horizon, with a number of new antifungal classes in late-stage clinical development. In this review, we describe the mechanisms of drug resistance employed by fungi and extensively discuss the most promising drugs in development, including fosmanogepix (a novel Gwt1 enzyme inhibitor), ibrexafungerp (a first-in-class triterpenoid), olorofim (a novel dihyroorotate dehydrogenase enzyme inhibitor), opelconazole (a novel triazole optimized for inhalation), and rezafungin (an echinocandin designed to be dosed once weekly). We focus on the mechanism of action and pharmacokinetics, as well as the spectrum of activity and stages of clinical development. We also highlight the potential future role of these drugs and unmet needs.
\end{abstract}

\section{Introduction}

The epidemiology of invasive fungal infections has changed over the last 2 decades, with broad-spectrum antifungal prophylaxis reducing prevalence and improving survival in patients with traditional risk factors. Selective pressure of antifungal prophylaxis (as well as advances in molecular testing) may be contributing to the emergence of formerly less common fungal pathogens, including rare yeasts and rare molds [1-5] that are often resistant to currently available classes of antifungal treatments. The diagnosis of these breakthrough infections can be challenging even under optimal circumstances, owing to insufficient sensitivities of current diagnostics [6]. At the same time, the emergence of Candida auris [7], as well as azole-resistant Aspergillus fumigatus [8], and cryptic species that are morphologically

Martin Hoenigl and Rosanne Sprute contributed equally to the article.

Martin Hoenigl

hoeniglmartin@gmail.com

Extended author information available on the last page of the article indistinguishable by classical methods within main Aspergillus sections [9] pose additional threats to our current antifungal armamentarium. In addition, new manifestations of disease in the intensive care population such as coronavirus disease 2019 (COVID-19)-associated aspergillosis [10-13] and mucormycosis $[14,15]$ are posing major problems because of extensive drug-drug interactions of antifungal drugs [16].

Despite the need for more antifungal drug options, no new classes of antifungal drugs have become available over the last two decades, and only one single new agent, isavuconazole [17], from a known antifungal class has been approved in the last decade. Fortunately, there are finally a number of new antifungal classes in late-stage clinical development. In this review, we discuss extensively the most promising drugs in development, including fosmanogepix (a novel Gwt1 enzyme inhibitor), ibrexafungerp (a first-in-class triterpenoid), olorofim (a novel dihyroorotate dehydrogenase enzyme inhibitor), opelconazole (a novel triazole optimized for inhalation), and rezafungin (an echinocandin designed to be dosed once weekly). We also highlight the potential future role of these drugs, the clinical trials currently evaluating them, and the spectrum of activity of these drugs. 


\section{Key Points}

The epidemiology of invasive fungal infections is changing, with new populations at risk and the emergence of antifungal resistance.

Despite the need for more antifungal drug options, no new classes of antifungal drugs have become available over the last two decades, but there is finally hope on the horizon, with a number of new antifungal classes in latestage clinical development.

In this review, we discuss the most promising antifungal drugs in development, focusing on the mechanism of action and pharmacokinetics, as well as the spectrum of activity and stages of clinical development.

We also highlight the potential future role of these drugs and unmet needs.

A literature search in July 2021 included: PubMed search for each compound name (old and new names) separately, searching the reference lists for additional studies, as well as search through abstracts presented at major scientific meetings in the field during the last 10 years.

\section{Antifungal Resistance}

Drug-resistant fungal pathogens have emerged and pose a significant clinical problem as treatment options are limited and infections may be associated with poor outcomes. Furthermore, some patients do not respond to antifungal therapy despite infection with a pathogen displaying susceptibility to the agent being used, a concept termed tolerance, which is discussed in more detail later. Thus, clinical therapeutic failure is a multifaceted phenomenon involving not only the mostly immunocompromised human host, but—although to a lesser extent-also the antifungal drug, and pathogenic fungi [18] and a combination of factors may contribute to therapeutic failure of antifungal drugs. Moreover, the concept of resistance laid out in the following will include mechanisms observed for three most predominant genera of fungi, namely Candida, Cryptococcus, and Aspergillus.

\subsection{Drug Tolerance Definition and Its Clinical Importance}

In the literature, the terms antifungal resistance and tolerance are frequently incorrectly used and resistance, resulting from a stable genetic change, needs to be distinguished from tolerance, resulting from unstable genomic, epigenomic, and physiologic changes. Both adaptive and transient resistance tolerates high drug concentrations beyond minimum inhibitory concentrations (MICs); this concept is different from classical resistance and is attributed to clinical failure and therefore we discuss this briefly (this concept is poorly studied in Aspergillus, while numerous studies have explored this phenomenon in both Candida and Cryptococcus). Once a fungus is exposed to a stressful environment, such as azole drugs, the most important priority for the cell is to survive. Therefore, employing transient and rapid mechanisms rewiring the transcriptomics and genomics landscape takes place, allowing a subpopulation of cells to grow slowly and survive at drug concentration beyond the MIC [19]. Because this phenotypic growth is slow, the tolerance measure is assayed at $48 \mathrm{~h}$, which is beyond the 24-h timepoint suggested by standard guidelines [19]. There is more attention to the concept of drug tolerance owing to the link between tolerance and azole therapeutic failure [19-23] and virulence [24]. Chromosomal duplications or aneuploidy may play a role in tolerance, as chromosomes housing the drug target and efflux pumps, and the resultant copy number variations are associated with a higher expression of the aforementioned genes. This may lead to more efficient expulsion of the drug out of the cells as well as allow for more available drug target to keep up with the excess of antifungal drugs, which ultimately provides a permissive state for cell survival [25-27]. These chromosomal changes are not stable and disappear following progressive passage on drug-free media [25-27]. Although tolerance and heteroresistance have many similarities regarding the underlying mechanisms and both survive at the concentration above the MIC $[25,27]$, it should be noted that the subpopulation size in tolerance $(5-90 \%)$ is larger than what has been noted for heteroresistance (much less than 1\%) [19]. Nonetheless, both phenomena are regarded as a window for gaining a stable drug resistance phenotype by allowing the tolerant minority subpopulation to survive in the presence of a high drug concentration [28-30]. Indeed, a new wave of studies has found the clinical importance of azole tolerance in therapeutic failure $[22,23,31]$ and future studies are underway to better understand the underlying mechanism, with the hope of identifying novel targets impairing this mechanism to thwart the emergence of resistance. Thus, tolerance is an emerging entity potentially affecting clinical success, which results from physiologic and unstable genomic changes.

\subsection{Drug Resistance Definition and Major Azole Resistance Mechanisms}

Unlike tolerance, drug resistance is a stable phenotypic growth beyond the MIC, which can be recorded at a 24-72-h 
time period and will not disappear after successive passage on drug-free media [19, 29]. Mechanisms involved in drug resistance have major overlaps among the three genera of fungi, but the mechanisms vary depending on the antifungal drug. Herein, our discussion includes the most predominant mechanisms believed to play a role against azoles.

Azoles are a class of antifungal drugs targeting one of the key enzymes involved in ergosterol biosynthesis, $14 \alpha$-lanosterol demethylase, which is encoded by ERG11 in Candida and Cryptococcus and CYP51A in Aspergillus [29, 32]. Acknowledging that resistance to azoles is multifactorial and there might be numerous factors functioning simultaneously with yet unidentified mechanisms, currently, it is thought that three major mechanisms are involved, namely drug target change, overexpression of drug target, and overexpression of efflux pumps [32].

1. Drug target changes due to mutations modulating the azole-binding site appear to be one of the most prevalent causes of azole resistance. Although several mutations have been found throughout the ERGII in yeasts, Y132F, corresponding to Y145F in Cryptococcus, and G458S, corresponding to G484S in Cryptococcus, are among the most prevalent amino acid substitutions, which result in therapeutic failure [7, 31, 33-36]. Interestingly, it has been shown that Candida parapsilosis isolates carrying $\mathrm{Y} 132 \mathrm{~F}$ are associated with a high mortality rate in addition to causing azole therapeutic failure $[36,37]$. More concerning is the emergence of azoleresistant $C$. parapsilosis isolates carrying $\mathrm{Y} 132 \mathrm{~F}$ in azole-naïve patients, possibly via environmental sources such as the hands of healthcare workers or devices routinely used in the clinic [37]. Of note, a growing number of studies show that the list of ERG11 mutations associated with azole resistance is expanding [37]. In A. fumigatus, the azole target has two copies, CYP51A and $C Y P 51 B$, and the occurrence of mutations in the former appear to be more accountable for resistance against mold-active triazoles [32]. Studies have shown that insertion of tandem repeats (TRs) with a length of 34bps and 46bps (TR34 and TR46) in conjunction with L98H and Y121F+T289A, respectively, are the most predominant mutations found that confer azole resistance [32]. Of note, A. fumigatus is intrinsically resistant to fluconazole due to a T301I amino acid substitution in CYP51A [38]. Moreover, numerous studies have also found azole-resistant isolates without mutations in the drug target, which has led to the discovery of new mechanisms and revealing the complexity of azole resistance $[24,25]$.

2. Overexpression of the drug target is another strategy employed to overcome azoles and employed by both yeasts and Aspergillus spp. Overexpression of the drug target results in the overproduction of ergosterol in the presence of the abundant azole drug, resulting in maintaining the membrane integrity. The occurrence of specific mutations in transcription factor/s regulating the drug target render them hyperactive, which is translocated into the nucleus, followed by binding to the promoter of the drug target and its overexpression as a result [39]. Such gain-of-function (GOF) mutations occur in UPC2 in Candida reported in numerous studies [40-42]. Expression of CYP51A in A. fumigatus, however, is regulated by the transcription factor SrBA [43]. Of note, the TR34/TR46 provide an extra binding site for SrbA and, therefore, strains carrying such TRs have an overexpressed CYP51A combined with mutations modulating the binding of the mold-active azoles [43].

3. Overexpression of efflux pumps expel the drug out of the cell and therefore help the fungus to mitigate the overwhelming concentrations of the azole drug. Efflux pumps belong to two major classes, including major facilitator superfamily (MFS), such as MDRl, and ATP-binding cassette (ABC) transporters, such as $C D R 1$ [19]. $A F R 1$ and $A F R 2$ correspond to $C D R 1$ and $M D R 1$ in Cryptococcus [44, 45]. In pathogenic yeasts, ABC and MFS transporters are mainly overexpressed by GOF mutations in TACl and MRRI, respectively [19, 30, 46]. Although azole resistance to ABC and MFS are well studied in Candida albicans [46], their functions and the importance of respective GOF mutations remain to be elucidated for other non-albicans Candida species. In $C$. glabrata, numerous GOF mutations in PDRI have been catalogued, which confer azole resistance, a higher virulence, as well as immunoevasion $[47,48]$. CDRlB is the main $\mathrm{ABC}$ transporter found in A. fumigatus, which is controlled by AtrR [49]. AtrR, in collaboration with SrbA, control the expression of CYP51A [50]. Of note, GOF mutations have not been described for AtrR and cataloging potential GOF mutations, followed by functional analysis to observe if they confer azole resistance, may potentially lead to the discovery of new pathways and/ or other players involving in azole resistance. Finally, ATRF is an MFS transporter, which has been found to be controlled by Yap 1 in Aspergillus flavus [51]. Therefore, its involvement in azole resistance and overexpression of ATRF in A. fumigatus is yet to be identified. It is noteworthy that these transcription factors play pivotal roles in virulence aside from drug resistance [52-54]. For instance, lines of evidence suggest that fluconazole resistant $C$. glabrata isolates carrying GOF mutations in PDRI are more immunoevasive and less identified by macrophages compared with their wild-type counterparts and paradoxically such isolates are more adherent to epithelial cells, which is mainly due to upregulation of EPA genes [52,53]. Although roles of 
such GOF mutations in SRBA and ATRR are not studied in A. fumigatus, current data suggest that both of these transcription factors play an important role in ergosterol biosynthesis, iron metabolism, nitrate assimilation, and adaptation to hypoxia. Therefore, mutant strains for lacking either or both of these genes have a significantly lower virulence relative to their wild-type backgrounds [54-56]. Clearly such data suggest that such transcription factors have pleiotropic biological functions, which extend beyond drug resistance, and play important roles in the survival of pathogenic fungi once exposed to various stresses, including adaptation to host-related niches.

\subsection{Resistance Mechanisms Against Echinocandins and Polyenes}

Resistance mechanisms against echinocandins appear to be straightforward, which does not require the involvement of a complex network. The major mechanisms found so far include the mutation in the hotspot regions of the catalytic subunit of $\beta-1,3$-D-glucan synthase, $F K S$ [31, 57-61]. It is noteworthy that in some cases mutations found in the hotspot regions does not confer in vitro resistance to echinocandins, while the infected patient experiences echinocandin therapeutic failure [62]. More recently, a change in the lipid composition of the microenvironment surrounding the $F K S$ gene is another proposed mechanism involved in echinocandin resistance [63]. Resistance against polyenes is relatively rare and mechanisms are poorly understood [32]. In most cases, the target is ergosterol, the mechanisms of action are ergosterol sequestration, and decreased membrane ergosterol and $E R G$ mutations are responsible for intrinsic or acquired resistance, including with $C$. auris and A. terreus [32].

\section{Antifungal Drugs in Clinical Development}

\subsection{Fosmanogepix/Manogepix}

Manogepix (APX001A, Amplyx Pharmaceuticals, Inc., San Diego, CA, USA; formerly E1210, Eisai Co., Ltd., Tokyo, Japan) is the active moiety of the N-phosphonooxymethyl prodrug fosmanogepix (APX001, formerly E1211). Following oral or intravenous administration, systemic phosphatases rapidly convert fosmanogepix to manogepix [64]. Recently, Pfizer, Inc. acquired Amplyx Pharmaceuticals.

\subsubsection{Mechanism of Action and Pharmacokinetics/ Pharmacodynamics}

Manogepix has a novel mechanism of action that targets glycosylphosphatidylinositol-anchored protein maturation through inhibition of the fungal enzyme Gwt1, an inositol acyltransferase that is essential for trafficking and anchoring mannoproteins to the fungal cell membrane and wall (Fig. 1) [65, 66]. Glycosylphosphatidylinositol-anchored mannoproteins serve as adhesions that allow fungi to adhere to mucosal and epithelial surfaces within the host prior to colonization and infection [67]. Some fungal adhesins and virulence factors are derived from glycosylphosphatidylinositol-anchored proteins [67-71]. The action of manogepix appears to be fungal pathogen specific, as it does not inhibit human inositol acylation by the closest mammalian ortholog, PIGW [64, 72].

Experimental mouse models of invasive candidiasis caused by different species of Candida, including $C$. albicans, $C$. glabrata, and $C$. auris, have demonstrated that the AUC/MIC is the pharmacokinetic/pharmacodynamic (PK/ $\mathrm{PD})$ parameter most closely associated with in vivo efficacy followed by maximum plasma concentration $\left(C_{\max }\right) / \mathrm{MIC}$ [73-75]. In one study, the median free fraction area under the curve (AUC)/MIC ratios associated with stasis ranged from 1.35 to 22.54, which correspond to total AUC/MIC targets of 675.5 to 11,270 [73]. Similarly, the AUC/MEC was the PK/PD target associated with efficacy in a neutropenic murine model of invasive aspergillosis [76]. Against wildtype A. fumigatus isolates and azole-resistant strains with CYP51A mutations, the median free fraction AUC/MEC ratio associated with a $1-\log$ reduction in fungal burden was 89.39 (total AUC/MEC 5258.2).

\subsubsection{Spectrum of Activity}

Manogepix has broad-spectrum activity against numerous pathogenic fungi (Fig. 2). Potent in vitro activity has been reported against Candida spp., including isolates of C. albicans, $C$. auris, and $C$. glabrata that are resistant to the azoles and echinocandins, Cryptococcus neoformans and $C$. gatti, Coccidioides spp., Aspergillus spp., including azole-resistant A. fumigatus, Fusarium spp., Scedosporium spp., Lomentospora prolificans, and other rare molds [65, 74-94]. Manogepix has been reported to lack in vitro activity against $C$. krusei and some of the Mucorales [65, 77], including variable activity against Rhizopus and Lichtheimia [89, 95], as well as Mucor and Cunninghamella [65, 89]. However, recent work has demonstrated activity in mouse models against Rhizopus delemar [96], and both in vitro and in vivo activity against Rhizopus arrhizus/oryzae, a frequent cause of mucormycosis in humans [97, 98]. In a mouse model, a combination of manogepix with liposomal amphotericin B showed a string synergistic effect reducing lung fungal burden and improving survival in invasive pulmonary aspergillosis, and reducing both lung and brain fungal burden and improving survival in mucormycosis [99]. It should be noted that, similar to the echinocandins, owing to its mechanism of action to compromise cell wall growth 
Fig. 1 Mechanism of action of novel antifungal drugs discussed in this review. $\mathrm{DHODH}$ dihydroorotate dehydrogenase

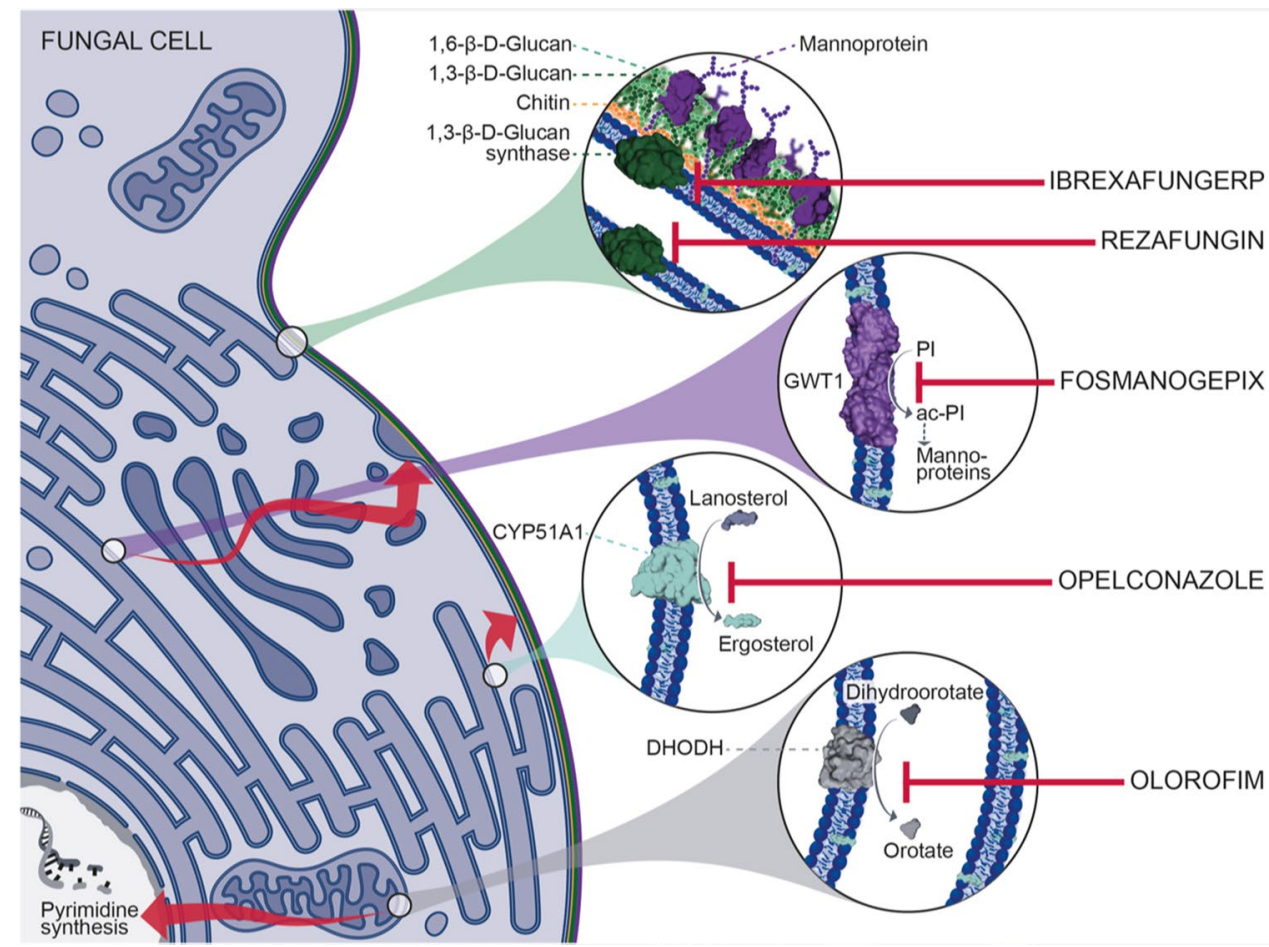

and hyphal elongation, the endpoint used for manogepix susceptibility against filamentous fungi is the minimum effective concentration (MEC). This is defined as the lowest concentration that results in morphologic changes to the fungus (i.e., short, stubby, abnormally branched hyphae) but not inhibition of growth as used for amphotericin B and the azoles $[64,100]$. The use of MEC leads to more stable and reproducible measurements in echinocandins. Manogepix has been shown to induce a similar morphological change to that of echinocandins in filamentous fungi [65]. The clinical relevance remains to be determined. The in vitro activity of manogepix has also translated into in vivo efficacy when fosmanogepix has been administered in experimental models of candidiasis, cryptococcosis, coccidioidomycosis, aspergillosis, fusariosis, scedosporiosis, and mucormycosis [73, $76,81,82,84,90,98,101]$.

\subsubsection{Clinical Studies}

3.1.3.1 Safety Four phase I clinical trials have been completed illustrating the safety and tolerability of fosmanogepix (Fig. 3; Table 1 of the Electronic Supplementary Material [ESM]). The initial study in humans was a randomized, double-blind, placebo-controlled, single ascending dose and multiple ascending dose-escalation study (NCT02956499). Six cohorts of eight healthy subjects per cohort were enrolled and randomized to receive 3-hour infusions of fosmanogepix or placebo. Those randomized to the single ascending dose study received $10-350 \mathrm{mg}$, while those ran- domized to multiple ascending doses received 50-600 mg once daily over 14 days duration. Fosmanogepix was well tolerated across all administered doses with no significant adverse events (AEs) [all described as mild, transient, and requiring no treatment]. Transient headache was the most frequently described AE [102]. There were no dose-limiting toxicities. The maximum tolerated dose was not determined in this study.

A second phase I study evaluated the safety, pharmacokinetics, bioavailability, and food effects of orally administered fosmanogepix (NCT02957929). Patients in this study were randomized to single intravenous doses of $200 \mathrm{mg}$ infused over 3 hours followed by single oral dosing (tablet) of 100 , 300 , and $500 \mathrm{mg}$ each separated by a 14-day washout period. They were also evaluated under fed and fasting conditions following a single oral dose of $400 \mathrm{mg}$. As in the initial phase I study, fosmanogepix was well tolerated across all studied doses with no clinically significant AEs observed. All subjects completed their assigned dosing regimen, and no dose-limiting toxicity was described. All AEs were, again, mild, transient, and required no specific treatment with headache that most frequently observed [103].

Additional phase I studies have also been performed: Safety and Pharmacokinetics of Intravenous and Oral APX001 in Patients With Acute Myeloid Leukemia (AML) and Neutropenia (NCT03333005), and A Drug-Drug Interaction Study of CYP3A4 Inhibition and Pan-CYP Induction on APX001 (NCT04166669). Although these results have not yet been published. 

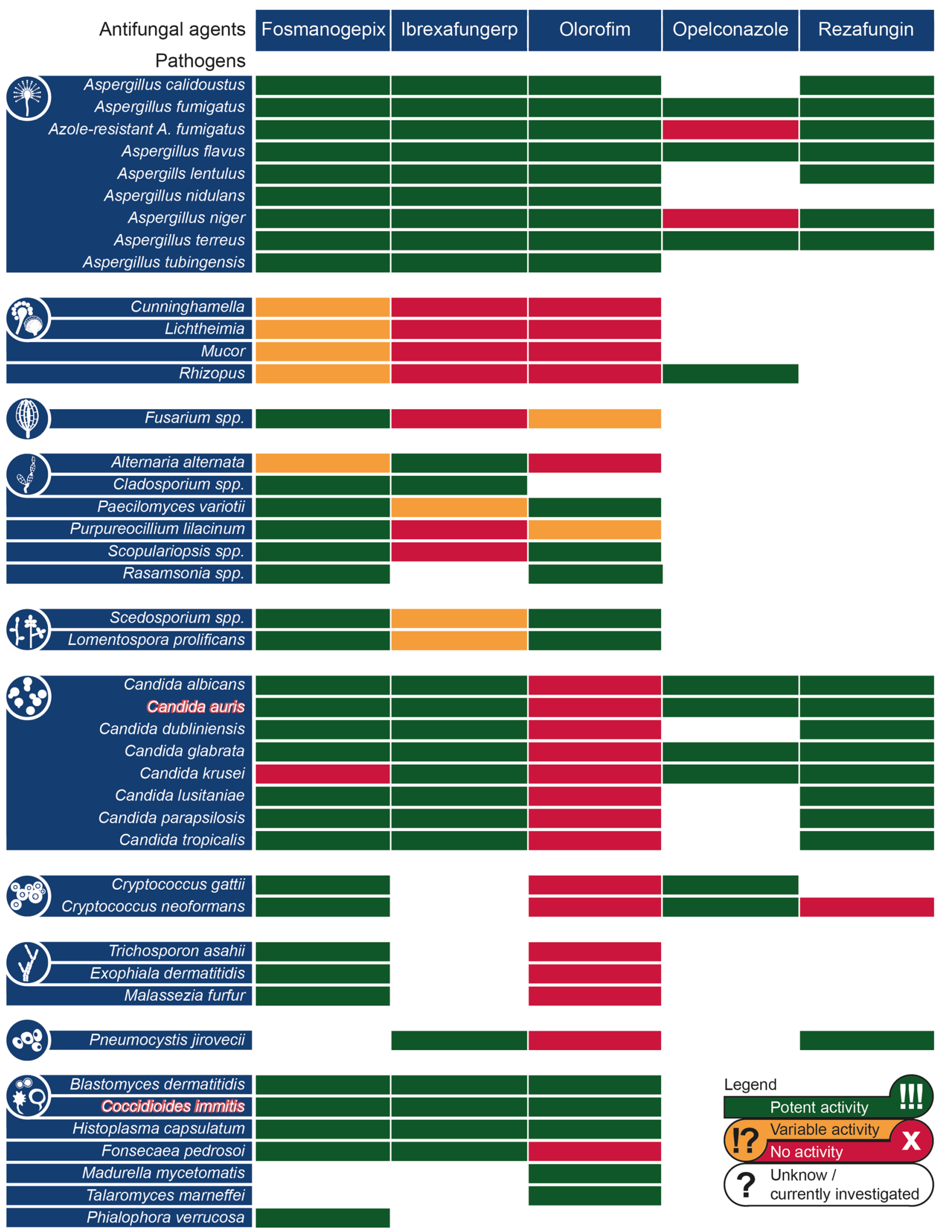

Antifungal agents

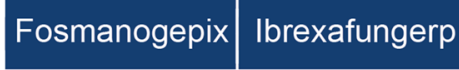

Olorofim

Opelconazole

Rezafungin

Fig. 2 Activity of the new antifungal drugs in the pipeline against most common fungal pathogens 


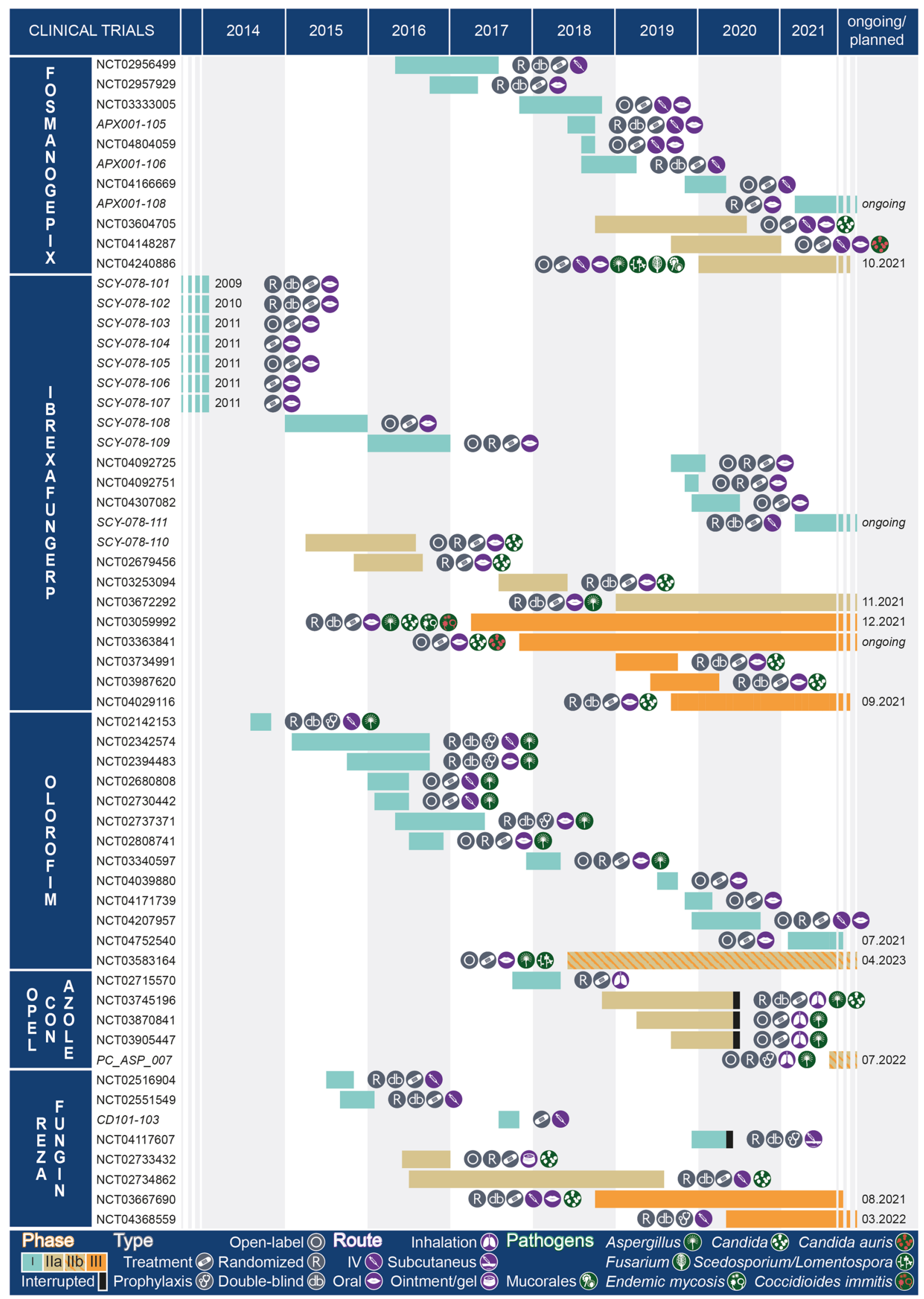

Fig. 3 Timetable of clinical trials evaluating fosmanogepix, ibrexafungerp, olorofim, opelconazole, and rezafungin. $I V$ intravenous 
In a phase II, multicenter, open-label, non-comparative, single-arm study of fosmanogepix for the treatment of invasive candidiasis in non-neutropenic patients, fosmanogepix was well tolerated with no treatment-related serious AEs or discontinuation in the modified intention to treat (MITT) group of 20 patients [104]. Patients with a recent diagnosis of candidemia defined as positive blood culture for Candida spp. within 96 hours prior to study entry, with $\leq 2$ days of prior antifungal treatment were eligible. Patients with neutropenia, C. krusei infection, or deep-seated Candida infections were excluded. Sixty-six of patients in this study had renal insufficiency and no worsening of renal function was observed. There was no evidence of drug-related nephrotoxicity and no dose adjustments were required suggesting that fosmanogepix may be safe in patients with impaired renal function [105]. Manogepix was also well tolerated in a cohort of nine intensive care unit patients with candidemia [106].

3.1.3.2 Efficacy In the above-mentioned phase II study, fosmanogepix was initiated as first-line therapy and patients were treated for up to 14 days [104]. Patients were prescribed fosmanogepix $1000 \mathrm{mg}$ intravenously twice a day for 1 day, then $600 \mathrm{mg}$ intravenously once daily for at least 2 days, followed by either $600 \mathrm{mg}$ intravenously once daily or $700 \mathrm{mg}$ orally once daily. The primary efficacy endpoint was the outcomes as adjudicated by an independent data review committee at the end of the study treatment. A successful outcome was defined as clearance of Candida spp. from blood with no additional antifungal therapy and survival at the end of study therapy. A success rate of $80 \%$ was observed in the MITT population. Negative blood cultures were observed after 2.4 days (mean) of fosmanogepix initiation, and efficacy was observed in isolates resistant to amphotericin B and/or anidulafungin. Among the patients who did not respond to therapy at the end of study therapy, $15 \%$ had persistently positive cultures, while one patient experienced an AE (leukopenia) and died because of bacterial sepsis. Overall survival at day 30 was $85 \%$ and none of the deaths was considered by the data review committee as related to fosmanogepix. In an interim analysis of nine intensive care unit cases with $C$. auris candidemia, treatment success and survival were achieved in 8/9 (89\%) of cases with one patient not responding to treatment [106].

3.1.3.3 Ongoing Studies An evaluation of fosmanogepix in the treatment of $C$. auris (APEX, NCT04148287) has recently been completed (Fig. 3; Table 1 of the ESM). This was a multicenter, open-label, non-comparative, single-arm study to evaluate the efficacy and safety of APX001 for the treatment of candidemia and/or invasive candidiasis caused by $C$. auris in patients aged 18 years and over with limited antifungal treatment options. Treatment was adminis- tered for a maximum of 42 days with a follow-up period of 4 weeks after the end of study therapy. An evaluation of fosmanogepix in the treatment of invasive aspergillosis and other rare molds (e.g., Scedosporium spp., Fusarium spp., and Mucorales fungi) is ongoing (AEGIS, NCT04240886). This study will enroll patients with limited or no treatment options because of documented or anticipated resistance, contraindication, intolerance, or a lack of clinical response to standard of care (SOC) antifungal therapy. The primary outcome is all-cause mortality at day 42 with key secondary outcomes of the global response at the end of study treatment or day 42 and all-cause mortality at day 84. Phase III trials for the treatment of invasive candidiasis and endemic fungal infections are planned.

\subsubsection{Future Role}

Fosmanogepix has been given fast track status by the US Food and Drug Administration (FDA) for invasive candidiasis, aspergillosis, scedosporiosis, fusariosis, mucormycosis, cryptococcosis, and coccidioidomycosis. Multiple ongoing studies will further define the role of this agent for the treatment of invasive fungal infections, although the favorable side-effect profile and activity against multiple pathogens with limited treatment options make this an attractive option and addition to the therapeutic armamentarium for a broad range of mold (variable activity only against Mucorales) and yeast infections (other than those caused by $C$. krusei) as well as endemic mycoses. The synergism with liposomal amphotericin B may increase attractiveness for using manogepix in combination for the most difficult-to-treat infections (Table 1).

\subsection{Ibrexafungerp}

Ibrexafungerp (MK-3118 and SCY-078; developed by Scynexis, Jersey City, NJ, USA) is a first-in-class oral glucan synthase inhibitor.

\subsubsection{Mechanism of Action and Pharmacokinetics/ Pharmacodynamics}

Ibrexafungerp is a triterpenoid antifungal inhibiting the biosynthesis of 1,3-beta-D-glucan in the fungal cell wall, as already known from echinocandins. Ibrexafungerp inhibits the 1,3-beta-D-glucan synthase enzyme and acts fungicidally on Candida spp. and fungistatically on Aspergillus spp. (Fig. 1) [107, 108]. Although the mechanism of action for ibrexafungerp and echinocandins is similar, the binding sites for both antifungal drugs are different with only a partial overlap. This results in very limited cross-resistance between ibrexafungerp and echinocandins [109-111]. Some Candida glabrata isolates with certain mutations in the FKS1 


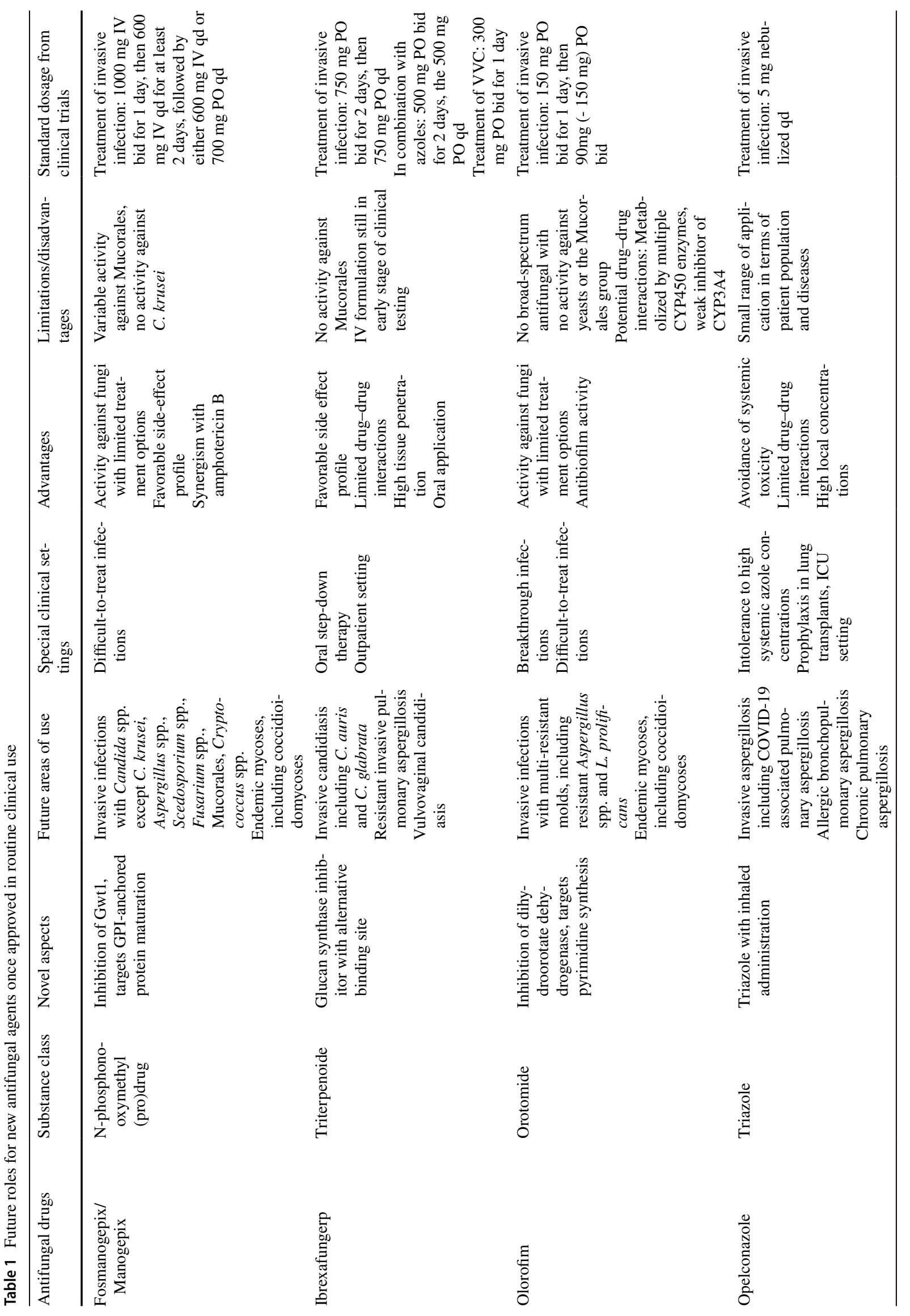




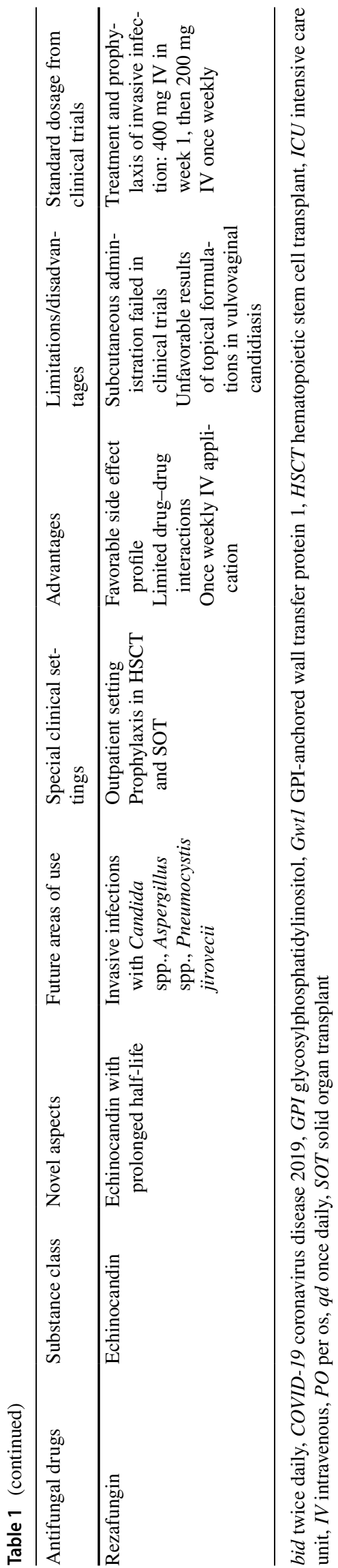

or $F K S 2$ genes, which are resistant to echinocandins, might also show resistance against ibrexafungerp. However, usually ibrexafungerp has potent activity against echinocandin resistant $C$. glabrata isolates with FKS mutations [111, 112] (see below for activity).

Ibrexafungerp is currently available as an oral formulation (an intravenous formulation is still in phase I developments) and has a good oral absorption with a bioavailability rate of 35-50\% and high protein binding of 99\% [113, 114]. Human data showed a peak plasma concentration within 4-6 $\mathrm{h}$ and a linear decline with a mean half-life of approximately 20-30 hours supporting a once-daily dosing strategy [115]. In ongoing studies, a once-daily dosage is used after 2 days of a twice-daily loading dose (FURI, NCT03059992). Although a high-fat meal increased the bioavailability, it delayed the median time to $C_{\max }$ from $4 \mathrm{~h}$ (fasted state) to 6 $\mathrm{h}$ [114]. Based on data from animal models, ibrexafungerp shows a high tissue penetration with the following tissueto-blood AUC ratios: spleen 54-fold; liver 50-fold; lung 31-fold; bone marrow 25-fold; kidney 20-fold; skin 12-fold to 18-fold; vaginal tissue nine-fold; and skeletal muscle fourfold, although tissue concentrations are known to not always infer site activity. Ibrexafungerp shows a minimal distribution to central nervous system tissues [116, 117]. In another study, ibrexafungerp accumulation was shown in vaginal tissue and fluid with a tissue concentration of twofold to fivefold higher than plasma concentrations and supporting the recent approval for the treatment of Candida vaginitis using a single-day 600-mg treatment (300 mg twice-daily dosage) [118]. Whereas penetration into the lens is poor, ibrexafungerp shows high concentrations in the uvea [116]. Ibrexafungerp is mainly eliminated via feces and is marginally recovered from urine (approximately 1\%) [116]. Ibrexafungerp is a CYP3A4 substrate and a reversible inhibitor of CYP2C8 and CYP3A4. However, the interaction with certain drugs and influence on drug concentrations is markedly weaker than observed with azoles. Ibrexafungerp has no effect on the maximum blood concentration of tacrolimus, a CYP3A4 substrate, with only a 1.4-fold increase in systemic exposure to tacrolimus. These findings support the coadministration of ibrexafungerp and tacrolimus without the need for an initial dose adjustment for tacrolimus [119]. Coadministration of strong CYP3A inducers (e.g., rifampin, St. John's wort) and ibrexafungerp should be avoided as ibrexafungerp might not reach sufficient drug concentrations, whereas coadministration of CYP3A inhibitors (e.g., ketoconazole or itraconazole) requires a dose reduction of ibrexafungerp (https://www.accessdata.fda.gov/drugsatfda docs/label/2021/214900s000lbl.pdf). In animal Pneumocystis models, ibrexafungerp reduced the fungal burden and improved survival. The results were comparable to trimethoprim-sulfamethoxazole standard therapy in one study and 
better for trimethoprim-sulfamethoxazole in another study [120-122].

\subsubsection{Spectrum of Activity}

Ibrexafungerp has in vitro activity against Aspergillus and Candida spp., with slightly weaker activity against $C$. lusitaniae and C. krusei compared with other Candida spp. (Fig. 2) [123]. Ibrexafungerp shows high activity against Candida isolates resistant to azoles (e.g., C. albicans, $C$. krusei, C. glabrata) but activity against echinocandinresistant Candida spp. is variable [123]. Overall, most echinocandin-resistant FKS mutants in Candida spp. are susceptible to ibrexafungerp, especially $C$. glabrata and C. auris isolates [112, 123-126], but some echinocandinresistant FKS mutants with the F641S, F649del, F658del, and F659del mutations also showed reduced susceptibility to ibrexafungerp [124, 127-129]. Ibrexafungerp is a potent inhibitor of Aspergillus spp. growth, including A. fumigatus, A. niger, A terreus, cryptic species [130], and azole-resistant strains $[108,109,127]$. Ibrexafungerp has no activity against Mucorales and Fusarium spp. but is very active against Alternaria and Cladosporium spp. [131, 132].

\subsubsection{Clinical Studies}

3.2.3.1 Safety Ibrexafungerp has already demonstrated good tolerability in single-dose and multiple-dose phase I/II studies, with non-serious AEs increased with exposed dosage and duration of therapy (Fig. 3; Table 2 of the ESM) [133]. The most common AEs are mild to moderate and due to gastrointestinal tract symptoms, including nausea, diarrhea, abdominal pain, and vomiting, that may be dose limiting [115, 134-136] [133]. In a phase II study, the safety of two dosing regimens of ibrexafungerp was investigated. Whereas one group including seven patients received 1000 $\mathrm{mg}$ of ibrexafungerp as a loading dose followed by $500 \mathrm{mg}$ daily, the second group containing six patients received a 1250 -mg loading dose followed by $750 \mathrm{mg}$ daily. As already observed in the phase I trial, most reported AEs were gastrointestinal tract symptoms; however, there was no distinct difference in AE frequency between the two groups and none of the AEs was considered severe enough to warrant the discontinuation of treatment [135]. Interim results of the ongoing phase III study show that oral ibrexafungerp was generally well tolerated, with the most common drugrelated AEs being mild-to-moderate diarrhea, nausea, and less frequently vomiting [137]. In contrast to several other orally available antifungal agents, ibrexafungerp did not cause a clinically relevant prolongation of the QTc interval in a group healthy volunteers [138]. Last, safety data for long-term treatment in humans are needed, although in preliminary animal data, 9 months of ibrexafungerp in dogs was well tolerated [139].

3.2.3.2 Efficacy In a randomized, phase II clinical trial investigating the efficacy, safety, and tolerability of different oral doses of ibrexafungerp in patients with invasive candidiasis, patients were randomized to receive oral step-down therapy after initial echinocandin treatment with either ibrexafungerp or fluconazole [135]. In the intention-to-treat analysis at the end of treatment, a favorable overall response was similar among the different study arms, with $71 \%$ of patients in the ibrexafungerp 500-mg group with a favorable outcome compared with $86 \%$ in the ibrexafungerp $750-\mathrm{mg}$ group and $71 \%$ in the fluconazole group. Of interest, six out of eight patients with Candida krusei or C. glabrata infections showed a favorable outcome when treated with ibrexafungerp. As oral therapy with azoles in these two species is challenging or not possible because of resistance, ibrexafungerp may represent a promising treatment option for such infections.

After completion of the phase II trial for patients with invasive candidiasis, there are currently multiple ongoing phase III trials. In an open-label, single-arm trial (FURI, NCT03059992), patients with invasive candidiasis who are refractory or intolerant to SOC antifungal treatment and patients with aspergillosis are being treated with oral ibrexafungerp ( $750 \mathrm{mg}$ twice daily for 2 days followed by 750 $\mathrm{mg}$ once daily; up to 180 days of treatment) with safety and efficacy as endpoints. This study is ongoing, although an interim analysis has been completed. Of 41 patients who were treated with oral ibrexafungerp for intra-abdominal infection, oropharyngeal or esophageal candidiasis, candidemia, or other forms of invasive candidiasis, $56 \%$ were considered as a partial or complete response and $15 \%$ as progressive disease [134]. Two patients with C. albicans and Candida tropicalis spondylodiscitis were also successfully treated with oral ibrexafungerp [140]. Given the need for a long treatment duration of Candida bone infections and the rising amount of azole-resistant and reduced susceptibility to Candida spp. [141], non-azole oral agents are needed to treat such infections in an outpatient setting. In the most recent interim analysis of 33 patients treated in 2019 and 2020 (each $45.5 \%$ with invasive candidiasis and mucocutaneous candidiasis, and 9\% with invasive pulmonary aspergillosis), complete/partial response or clinical improvement was observed in $70 \%$, with $21 \%$ stable disease as a global response at the end of treatment $(9 \%$ unable to determine) [137]. A second single-arm, open-label, phase III trial (CARES, NCT03363841) is evaluating patients with $C$. auris infections (invasive candidiasis and candidemia) with oral ibrexafungerp (add on to ongoing intravenous therapy or ibrexafungerp monotherapy) as emergency treatment. Among the first ten patients enrolled were eight patients 
(80\%) who had a complete response, one patient died of other causes, and one patient outcome was indeterminate [142].

In addition, ibrexafungerp has been studied in patients with vulvovaginal candidiasis (VVC). In a randomized, multicenter, evaluator-blinded study of 96 patients with moderate or severe VVC, patients were randomized to receive oral ibrexafungerp for 3 days, ibrexafungerp for 5 days, or fluconazole [143]. In the intention-to-treat analysis, both ibrexafungerp treatment regimens showed similar efficacy with a clinical cure rate of $78 \%$ in the ibrexafungerp-treated patients vs $66 \%$ in the patients receiving fluconazole by day 24 following randomization. Based on two phase III trials, VANISH-303 and VANISH-306, ibrexafungerp was recently approved by the FDA for oral use in adult and post-menarchal pediatric female patients with VVC with a recommended dose of $300 \mathrm{mg}$ twice daily for 1 day (Brexafemme ${ }^{\circledR}$ ). In the multicenter, randomized, double-blinded VANISH-303 trial [144, 145], patients in the USA were randomized in a 2:1 ratio to treatment with either ibrexafungerp $600 \mathrm{mg}$ once daily or placebo. The VANISH-306 trial has the same design as the VANISH-303 trial but allowed patient inclusion not only in the USA but also in Bulgaria. The primary endpoint for both studies was clinical cure (resolution of all signs and symptoms) at the test-of-cure (TOC) visit (day $11 \pm 3$ days). Overall, 290 patients (190 in the ibrexafungerp group and 100 in the placebo group) and 278 (189 in the ibrexafungerp group and 89 in the placebo group) were included in the modified intentto-treat analysis (requiring isolation of yeasts at baseline) in the VANISH-303 and VANISH-306 study, respectively. In both trials, ibrexafungerp was superior to placebo regarding clinical cure at the TOC visit [risk ratio 1.70 (95\% confidence interval 1.2-2.5) for the VANISH-303 trial and risk ratio 1.35 (95\% confidence interval 1.06-1.73) for the VANISH-306 trial]. In addition, ibrexafungerp was superior for secondary endpoints including mycological eradication at TOC, overall success at TOC, clinical improvement at TOC, and clinical cure at follow-up [145].

3.2.3.3 Ongoing Studies The FURI and CARES phase III trials are currently ongoing (Fig. 3; Table 2 of the ESM). Data on the efficacy and safety of ibrexafungerp for the treatment of mold infections are lacking, apart from three cases with invasive pulmonary aspergillosis. In a recent in vitro and in vivo animal model study, combination ibrexafungerp and isavuconazole resulted in a synergistic in vitro interactions and the combination of ibrexafungerp $(7.5 \mathrm{mg} /$ $\mathrm{kg}$ bodyweight daily) plus isavuconazole (40 mg/kg bodyweight daily) not only reduced pulmonary fungal burden in a neutropenic rabbit model but also prolonged survival [146]. Currently, there is an ongoing phase II, multicenter, randomized, double-blind trial evaluating the efficacy and safety of ibrexafungerp as combination therapy in patients with invasive pulmonary aspergillosis (SCYNERGIA, NCT03672292). In addition, the role of ibrexafungerp for the prevention of VVC is currently being investigated in a phase III trial (CANDLE, NCT04029116).

\subsubsection{Future Role}

Ibrexafungerp was approved by the FDA for the treatment of VVC on 1 June, 2021, with other approvals likely to follow soon. Echinocandins are considered the standard treatment for invasive Candida infections; however, they are only available as intravenous formulations and oral alternatives with similar activity are often lacking. A recent multinational European study showed that hospital stay was prolonged because of parenteral therapy in $21 \%$ of cases with invasive Candida infections [147] owing to a lack of a reliable oral alternative. Ibrexafungerp will fill the gap and will likely be used for primary and oral step-down therapy of invasive Candida infections. Ibrexafungerp will also fill the gap of being an oral antifungal drug with limited contraindications, resistance, or potential drug-drug interactions as well as high tissue penetration and broad and fungicidal activity particularly against yeasts including $C$. auris. For invasive pulmonary aspergillosis, it may serve a role as therapy for resistant cases, particularly in combination therapy with an azole or amphotericin B lipid formulations (Table 1).

\subsection{Olorofim}

Olorofim (F901318, developed by F2G, Inc., Manchester, $\mathrm{UK})$ is a member of a novel class of antifungal drugs named ortomides.

\subsubsection{Mechanism of Action and Pharmacokinetics/ Pharmacodynamics}

Olorofim inhibits fungal growth through inhibition of the fungal dihydroorotate dehydrogenase enzyme involved in pyrimidine synthesis, and without any significant crossreactivity with the human dihydroorotate dehydrogenase, limiting the compounds on target drug toxicity (Fig. 1) [117, 148, 149]. The compound itself has poor water solubility and is highly protein bound, but excellent tissue distribution including the kidney, liver, lung, and the brain (at lower levels). Oral dosing is $45 \%$ bioavailable. Susceptible fungi exhibit time-dependent killing effect after dosing. Olorofim is metabolized by multiple CYP450 enzymes including CYP3A4 and is thus susceptible to strong CYP3A4 inhibitors and inducers [117]. Olorofim does not appear to have any effect on the CYP450 enzymes, and has a low potential for drug interactions $[117,150]$. 


\subsubsection{Spectrum of Activity}

Olorofim is not a broad-spectrum antifungal; however, it does have significant novelty and a spectrum of activity that will be relevant in the future. Olorofim exhibits no activity for yeast, Exophiala dermatitidis [151], or the Mucorales group [152] of thermally monomorphic molds, and also no activity against Alternaria alternata [153], but does have activity against several clinically important groups of fungi, including dimorphic molds (e.g., Histoplasma and Coccidioides spp.), the hyaline hyphomycetes (e.g., Aspergillus spp.), and dematiaceous molds (e.g., Scedosporium spp.) (Fig. 2) [117, 154-156]. Olorofim was highly active in vitro and in vivo against central nervous system coccidioidomycosis in a mouse model [157]. Olorofim has shown excellent in vitro activity against both mold and yeast phases of Talaromyces marneffei [158], and also against Madurella mycetomatis, the main causative agent of eumycetoma [159] as well as dermatophytes including Trichophyton spp. [153]. By virtue of its novel target enzyme and mechanism of action, olorofim has activity against isolates that are resistant to current treatment options, including several species that can be resistant to all commercially available antifungal drugs such as Scedosporium apiospermum, L. prolificans, Rasamsonia spp., Penicillium spp., and Scopulariopsis spp. (including Scopulariopsis brumptii) [117, 151, 153, 160-164]. Olorofim also maintains good activity against azole resistant $A$. fumigatus isolates [151, 165], including CYP51A active mutants and hard-to-treat cryptic species of Aspergillus, such as A. nidulans, A. tubingensis, A. lentulus, and A. calidoustus [152, 154, 166, 167]. Of the common hyaline molds, olorofim exhibits overall good activity against Fusarium solani species complex and Fusarium oxysporum, with some isolates demonstrating MICs up to $4 \mathrm{mg} / \mathrm{L}$, while others demonstrate low MICs $[152,168]$.

\subsubsection{Clinical Studies}

3.3.3.1 Safety There is currently not much known about the adverse-effect profile of olorofim as clinical studies are ongoing, although it appears to be safe. Olorofim was well tolerated in a phase I study of once-daily oral olorofim dosed for 10 days in healthy volunteers (Fig. 3; Table 3 of the ESM). No serious AEs were reported, although two participants experienced nausea and diarrhea and one experienced dizziness [169]. In a multiple-dose study of 40 healthy male volunteers (NCT02142153), there also were no serious AEs reported [170], with a frequency of non-serious AEs as follows: musculoskeletal pain in 0/30 participants in the olorofim group vs $1 / 10$ in the placebo group; paresthesias or headache in $2 / 30$ in the olorofim group vs $0 / 10$ in the placebo group; epistaxis in $2 / 30$ in the olorofim group vs $1 / 10$ in the placebo group; and eczema in 1/30 participants in the olorofim group vs 0/10 in the placebo group [171]. Last, in the olorofim group, infusion-related reactions including dizziness (67\%), infusion-site pain (44\%), and phlebitis (39\%) were reported; each of these AEs was reported in $17 \%$ of participants in the placebo group [170].

In November of 2019, olorofim was granted Breakthrough Therapy designation for the treatment of invasive mold infections in patients with limited or no treatment options by the FDA and again in October 2020 for the treatment of central nervous system coccidioidomycosis refractory to SOC therapy. In addition, olorofim was granted orphan drug designation in March 2020 for the treatment for invasive aspergillosis, L. prolificans, and Scedosporium spp. infections followed by coccidioidomycosis in June of 2020. Last, it was granted Qualified Infectious Disease Product designation in June of 2020 for the treatment of invasive aspergillosis, invasive scedosporiosis, invasive lomentosporiosis, coccidioidomycosis, invasive scopulariopsis, and invasive fusariosis.

3.3.3.2 Efficacy There are no published reports describing the clinical efficacy of olorofim, although there are a number of case reports that have been presented in abstract form at conferences. In one case, a 56-year-old woman with acute T-cell lymphoblastic leukemia underwent HyperCVAD and developed disseminated $L$. prolificans infection involving her bloodstream, lungs, and aortic valve, and she developed L4/5 vertebral osteomyelitis and endophthalmitis. She underwent surgical debulking of the spine and did not respond to dual antifungal therapy with voriconazole plus terbinafine. After 11 months from the onset of infection, she was started on olorofim monotherapy (loading dose of $180 \mathrm{mg}$ followed by $60 \mathrm{mg}$ twice daily followed by 90 mg twice daily). After 6 months, she developed radiologic improvement with decreased uptake on a positron emission tomography scan and clinically improved with weight gain and stabilization of vision. She ultimately received a year of oloforim without any adverse effects [172]. In another case report, a 49-year-old woman developed disseminated $L$. prolificans following a right breast implant, which spread to her soft tissue, ribs, and sternum. Despite implant removal, repeated debridement, hyperbaric oxygen therapy, and multiple antifungal treatment including voriconazole plus terbinafine, miltefosine, posaconazole, and anidulafungin, her infection persisted. She was started on olorofim $(60 \mathrm{mg}$ twice daily followed by $90 \mathrm{mg}$ twice daily followed by 120 mg twice daily) for a total of 322 days. This was well tolerated and eventually the surgical site healed [173]. Last, a 45-year-old man with insulin-dependent diabetes mellitus developed disseminated coccidioidomycosis infection with central nervous system involvement. He did not respond to antifungal treatment with fluconazole, voriconazole, itraconazole, liposomal amphotericin B plus posaconazole, and 
posaconazole plus micafungin and continued to clinically deteriorate. After 8 months, he was switched to posaconazole plus olorofim (120 mg twice daily). Radiographic and clinical improvement was noted and his Coccidioides complement fixation titer decreased from 1:128 to 1:32 within 5 months [174].

3.3.3.3 Ongoing Studies Currently, olorofim is being evaluated in an open-label, single-arm, phase IIb clinical trial for the treatment of invasive mold infections with limited treatment options (FORMULA-OLS, NCT03583164), including infections due to $L$. prolificans, Scedosporium spp., Aspergillus spp., Coccidioides spp., and other invasive molds with resistance to commercially available antifungal drugs or for patients who are not responding to traditional therapy (Fig. 3; Table 3 of the ESM) [175].

\subsubsection{Future Role}

Olorofim will have a central role in the treatment of multiresistant mold infections, including azole-resistant aspergillosis. In particular for L. prolificans, where other treatment options are scarce because of pan resistance [2, 4, 176, 177], olorofim may present a "breakthrough" treatment option [151], as it is not only associated with very low MICs but also has antibiofilm activity [178]. In addition, olorofim will have a very important role in the treatment of endemic mycoses, particularly coccidioidomycoses, but also infections caused by T. marneffei [158], and Madurella mycetomatis (Table 1).

\subsection{Opelconazole}

Opelconazole (PC945, developed by Pulmocide Ltd., London, UK) is a first-in-class inhaled antifungal drug from the class of broad-spectrum triazoles.

\subsubsection{Mechanism of Action and Pharmacokinetics/ Pharmacodynamics}

Opelconazole is a novel antifungal triazole that was designed and optimized for inhalation via commonly available nebulizers [179]. After inhalation, opelconazole shows efficacy primarily in the lungs (systemic concentrations are minimal), making it a promising agent for treating pulmonary aspergillosis in non-neutropenic patients without disseminated infection. Its primary mechanism of action is familiar and comparable to established azoles, as opelconazole also contains the typical heterocyclic triazole scaffold. By inhibiting lanosterol 14 $\alpha$-demethylase (CYP51A1), lanosterol conversion to ergosterol is inhibited, leading to a reduction in ergosterol synthesis and hence dysfunction of fungal membrane structure, preventing further growth (Fig. 1)
[180]. Recent in vitro and in vivo studies have shown distinctive pharmacological characteristics, differentiating from commonly used azoles. Chemical and physical attributes of opelconazole, namely increases in lipophilic compounds and micronized drug particles, result in high local concentrations, prolonged lung retention, slow absorption form the lung, and as a consequence, low plasma concentrations [179].

These findings are promising given the low potential for systemic adverse drug effects and drug-drug interactions. Additionally, some data suggest cellular persistence of opelconazole in local immune and epithelial cells, which is potentially valuable in terms of use in prophylaxis or enhancement of antifungal activity $[181,182]$.

\subsubsection{Spectrum of Activity}

Opelconazole shows broad-spectrum antifungal activity against yeasts and molds, including Candida spp. including C. albicans, C. glabrata and C. krusei, C. neoformans, and gattii and Aspergillus spp. including A. fumigatus, $A$. carbonarius, and A. flavus. For the emerging yeast $C$. auris, which will potentially constitute a pathogen with limited treatment options because of its multi-drug-resistant nature, potent inhibition was noted [14]. Remarkable potency was also seen against Rhizopus arrhizus/oryzae (Fig. 2) [180]. Opelconazole has shown in vitro superiority over posaconazole, itraconazole, and voriconazole in azole-susceptible and azole-resistant strains of A. fumigatus. Against 96 clinically isolated A. fumigatus strains, opelconazole showed 2.5-fold more potency compared with voriconazole and comparable potency for posaconazole [180]. CLSI methods, as well as the EUCAST microdilution method, validated superiority over voriconazole. In immunocompromised mice, comparable in vivo findings were seen [180]. Opelconazole lacks activity against Aspergillus niger, Lichteimia corymbifera, Fusarium graminearum, Penicillium chrysogenum, and Penicillium citrinum. [180]. Remarkable synergistic effects were reported in an in vitro human alveolus bilayer model when opelconazole was administered apically combined with basolateral posaconazole or voriconazole, illustrating an in vitro model of concomitant topical lung and systemic therapy [182]. Interestingly, these effects were not present when combining posaconazole or voriconazole locally and systemically, highlighting the superiority of opelconazole pharmacokinetics regarding local drug activity, compared with established azoles [182].

\subsubsection{Clinical Studies}

3.4.3.1 Safety To date, a small number of phase I and phase IIa studies have investigated clinical safety (Fig. 3; Table 4 of the ESM). Cass et al. analyzed safety profiles in 29 sub- 
jects, of whom 20 were healthy and nine had mild asthma. Following inhaled single doses of $5 \mathrm{mg}$ in the mild asthma cohort, single doses between 0.5 and $10 \mathrm{mg}$ in healthy cohort one and repeated doses of $5 \mathrm{mg}$ for 7 days in healthy cohort two [179], the geometric mean plasma $C_{\max }$ after the 5-mg single dose did not significantly differ between healthy subjects and subjects with mild asthma. A dose-proportional increase in the AUC from 0 to $24 \mathrm{~h}$ and $C_{\max }$ was found between dosages of $0.5 \mathrm{mg}$ to $10 \mathrm{mg}$. However, oral inhalation of PC945 resulted in low systemic exposure as demonstrated by a mean plasma $C_{\max }$ of $0.951 \mathrm{ng} / \mathrm{mL}$ following 5 -mg doses once daily for 7 days, suggesting that it will have a negligible systemic antifungal effect and a much lower drug-drug interaction risk than orally administered triazole antifungal drugs. In addition to mild-to-moderate AEs (e.g., cough, throat tightness, headache, nausea, fatigue), which all resolved by the end of the study, no severe events were observed. Compared with the placebo cohort, no significant differences in spirometry values, electrocardiogram recording, vital signs, or mean laboratory values were noted. In the mild asthma cohort, no significant decrease in lung function or triggering of bronchospasm was identified [179]. Inhibitory effects were found on CYP3A4/5 substrates but opelconazole showed no interaction with other CYP isoforms [179]. These first in-human clinical findings underline that pharmacokinetic (PK) properties of PC945 and drug administration via inhalation will likely lead to a superior safety profile compared with systemic application.

3.4.3.2 Efficacy The efficacy of inhaled opelconazole was investigated in A. fumigatus-infected neutropenic mice, focusing on fungal burden, dynamics of biomarkers (BALF, Serum GM), and fungus-related inflammatory response after various treatment regimens, including for prophylaxis. Results showed strong inhibition of fungal burden in lung tissue, substantial decrease of GM, and suppression of inflammatory cell accumulation in dose-dependent relations [183]. Opelconazole showed marked superiority to voriconazole and posaconazole when evaluating the aforementioned parameters and very high dosages of voriconazole and posaconazole were needed to achieve similar results, once more highlighting that the strength of opelconazole is sustained lung retention and persistent antifungal activity [183].

3.4.3.3 Ongoing Studies In an ongoing study, opelconazole has shown promising results when used as part of its compassionate use program, acting as a last resort in patients who have not responded to standard antifungal therapies (Fig. 3; Table 4 of the ESM). Of nine patients treated, positive clinical results were observed in eight. An open-label randomized phase IIb study will start in 2021, focusing on the safety and tolerability of opelconazole prophylaxis and treatment of invasive aspergillosis in lung transplant recipients.

\subsubsection{Future Role}

Currently available antifungal agents have been approved for oral or intravenous application, but the emergence of primary airway invasive aspergillosis in non-traditional risk groups has lately increased the interest in inhaled antifungal agents. In order to achieve local efficacy, high systemic concentrations, which are frequently associated with treatment limiting complications, are required. Thus, other modes of delivery highlight unmet needs in antifungal treatment. Topical or inhaled administration may maximize local efficacy while avoiding systemic toxicity, decreasing the need for high drug concentrations and potential toxicity. Established drugs were tried to be repurposed but do not meet PK/pharmacodynamic $(\mathrm{PD})$ properties and airway tolerability allowing sufficient local treatment. Particularly for combination therapy approaches in invasive aspergillosis, opelconazole would be a new therapeutic option of potential great value by achieving high local concentration without systemic drug concentrations and toxicity, specifically attractive for primarily airway invasive aspergillosis in non-neutropenic patients, including those with COVID-19-associated pulmonary aspergillosis [10, 184]. Other roles may include antifungal prophylaxis after lung transplantation and in the intensive care unit setting or also in other settings where mold-active prophylaxis is not yet firmly established (e.g., induction chemotherapy for acute lymphocytic leukemia or stem cell transplantation early phase) as well as combination therapy with a systemic antifungal agent for cases with angioinvasive pulmonary aspergillosis (Table 1).

\subsection{Rezafungin}

Rezafungin (formerly SP3025 and CD101; Cidara Therapeutics, San Diego, CA, USA) is considered the first member of second-generation echinocandins with enhanced PK/PD pharmacometrics [185]. This novel drug is currently investigated in two phase III trials (ReSTORE, NCT03667690 and ReSPECT, NCT04368559) to assess its therapeutic use in candidemia and other invasive candidiasis and its potential to prevent invasive fungal disease (IFD).

\subsubsection{Mechanism of Action and Pharmacokinetics/ Pharmacodynamics}

Rezafungin was designed to optimize PK properties and avoid hepatotoxicity by reducing degradation while maintaining the potent antifungal activity and safety profile of the echinocandin class [186]. Echinocandins are lipopeptide antifungal drugs that have a cyclic depsipeptid core and 
an N-linked acyl lipid side-chain [187] that is considered essential for the antifungal activity [188]. Consistent with other echinocandins, the antifungal activity of rezafungin is carried out by inhibition of the cell-wall enzyme complex $\beta$-1,3-D-glucan synthase (Fig. 1) [188]. Rezafungin is a chemical analog to anidulafungin with a similar alkoxy triphenyl moiety but a distinct structural modification at the C5 ornithine hemiaminal of the cyclic core, which is replaced by a choline aminal ether [189]. This modification results in a considerably longer half-life, as non-enzymatic chemical degradation occurs on the hemiaminal of anidulafungin [189]. Rezafungin is stable to biotransformation in liver microsomes or hepatocytes, reducing the risk of hepatotoxicity, similar to other echinocandins [186]. As with other echinocandins, in vitro cytochrome inhibition studies suggest minimal interaction with CYP450 enzymes [186].

Pharmacokinetic results from two phase I dose-escalation studies (NCT02516904 and NCT02551549) have shown a mean half-life of approximately 80 hours after the first dose and 150 hours after the second or third dose indicating linear pharmacokinetics [190]. This allows rezafungin to be administered at extended intervals, such as once weekly. Mean plasma $C_{\max }$ and AUC have been shown to increase in proportion to dose [190]. Renal clearance plays a minor role in the excretion of rezafungin with fractions excreted $<1 \%$ at all dose concentrations [190]. In healthy subjects, no relevant PK interactions were monitored when dosing rezafungin concomitantly with several probe drugs [191].

\subsubsection{Spectrum of Activity}

In vitro susceptibility testing of rezafungin has been performed in several studies for wild-type and resistant fungal isolates using reference EUCAST and CLSI methodologies, which have been shown to have an excellent level of concordance for testing rezafungin [192]. The activity of rezafungin against Candida spp. is comparable to that of other members of the echinocandin class (Fig. 2) [192]. Candida albicans appears to be very susceptible to rezafungin, as the majority of isolates were inhibited by $\leq 0.125 \mu \mathrm{g} / \mathrm{mL}$ in different laboratories using CLSI and EUCAST methods [193, 194]. Candida dubliensis, C. fabianii, C. glabrata, C. inconspicua, C. kefyr, C. krusei, C. lipolytica, C. pulcherrima, C. sojae, and $C$. tropicalis were also inhibited by MIC values $\leq 0.125$ $\mu \mathrm{g} / \mathrm{mL}$ and were susceptible to other echinocandins using epidemiological cut-off value (ECV) interpretive criteria [194, 195]. For C. lusitaniae and C. auris isolates, MICs were $0.25 \mu \mathrm{g} / \mathrm{mL}$ by CLSI methodology [195]. Minimum inhibitory concentrations of $0.5 \mu \mathrm{g} / \mathrm{mL}$ were found for C. metapsilosis and $1 \mu \mathrm{g} / \mathrm{mL}$ for C. orthopsilosis and C. guilliermondii [195]. Candida parapsilosis was the least susceptible organism with MICs up to $4 \mu \mathrm{g} / \mathrm{mL}$ [194]. Intrinsically elevated MICs for $C$. parapsilosis are similarly described for other echinocandins and attributed to a polymorphism in the $F K S$ gene of $C$. parapsilosis [196]. However, consistent treatment failures have not yet been demonstrated [196]. For isolates harboring $F K S$ mutations, elevated rezafungin MICs were described with a similar or slightly better activity against mutant Candida spp. compared to other echinocandins [193, 197]. In summary, rezafungin demonstrates potent in vitro activity against most wild-type and azole-resistant Candida spp., including C. auris. Rezafungin also has potent activity vs common dermatophytes (e.g., Trichphyton mentagrophytes, T. rubrum, Microsporum gypseum, Epidermophyton floccosum).

Rezafungin has also demonstrated efficacy for the treatment of invasive candidiasis in vivo, with potent activity against $C$. albicans, including azole-resistant $C$. albicans, C. glabrata, and C. parapsilosis strains in murine models of immunocompromised disseminated candidiasis [198, 199]. For Aspergillus spp., rezafungin MEC ranges of $\leq 0.015-0.125 \mu \mathrm{g} / \mathrm{mL}$ and $\leq 0.015-2 \mu \mathrm{g} / \mathrm{mL}$ were reported against $A$. fumigatus wild-type and azole-resistant species, respectively [200]. Against A. flavus, A. niger, and A. terreus, rezafungin was shown to be active with MECs of $\leq 0.008-0.03 \mu \mathrm{g} / \mathrm{mL}$ [192]. Rezafungin also has activity against cryptic species, including A. calidoustus, A. lentulus, A. thermomutatus, and A. udagawae [200]. The activity of rezafungin against Aspergillus spp. is therefore comparable to other echinocandins. In a disseminated infection mouse model of aspergillosis caused by A. fumigatus, similar survival rates were shown with rezafungin compared to amphotericin B treatment [199]. In vivo efficacy has also been demonstrated in an murine model of disseminated aspergillosis caused by an azole-resistant $A$. fumigatus isolate harboring a $\mathrm{TR}_{34} / \mathrm{L} 98 \mathrm{H}$ CYP51A mutation [201]. Additional studies are warranted to determine whether this preclinical research translates to clinical experience and if rezafungin has the potential to prevent and treat infections caused by Aspergillus spp.

Echinocandins are not commonly used to treat or prevent Pneumocystis jirovecii infections. However, rezafungin has been shown to prevent $P$. murina infection in an immunosuppressed mouse model of Pneumocystis jirovecii pneumonia. A significant decrease in the number of trophic nuclei and reduced count of cystic forms have been demonstrated in the rezafungin-treated groups, with a comparable efficacy to the active control trimethoprim-sulfamethoxazole [202].

Like other echinocandins, rezafungin is inactive against non-Aspergillus molds, Cryptococcus, Trichosporon, and Rhodotorula isolates with MICs $>8 \mu \mathrm{g} / \mathrm{mL}[188,193]$. Overall, rezafungin exhibits broad in vitro potency against fungal pathogens comparable to that of other echinocandins. To date, in vitro data are lacking for most of the rare and emerging molds and yeasts. 


\subsubsection{Clinical Studies}

3.5.3.1 Safety Phase I studies of rezafungin consisted of two randomized, double-blind, placebo-controlled doseescalation studies with doses up to $400 \mathrm{mg}$ once weekly for 3 consecutive weeks (NCT02516904 and NCT02551549, Fig. 3; Table 5 of the ESM) [190]. Safety was assessed by the number of clinically significant AEs in healthy adults. The majority of AEs reported were mild, with chest discomfort, constipation, flushing, nausea, and myalgia being frequently reported. A tendency toward higher rates of AEs, including transfusion reactions, was observed in the group receiving the highest dose of rezafungin. All AEs were transient and resolved completely. No severe or serious AEs, withdrawals because of AEs, or deaths were reported. Furthermore, no safety issues related to laboratory results, physical examination, or vital signs were reported. Thus, rezafungin was shown to have a favorable safety profile at once-weekly doses of $400 \mathrm{mg}$.

In addition, a randomized, double-blind, phase I study was conducted in healthy volunteers to evaluate cardiac effects of single doses of intravenous rezafungin [203]. For echinocandins, data on QT prolongation among echinocandins are limited. For anidulafungin, QT prolongation is reported as an AE [204]. Rezafungin infusions did not prolong QT/QTc interval at doses up to $1400 \mathrm{mg}$, and there was no apparent effect on repolarization or QRS duration in a 12-lead electrocardiogram. Echocardiograms showed no change in ejection fraction or other cardiac parameters compared with baseline and an increase in PR interval was seen in the 1400-mg dose group, but was considered not clinically relevant. No serious AEs were reported, and no subjects discontinued the study because of an AE. The 1400$\mathrm{mg}$ dose is six-fold higher than expected to be achieved at therapeutic steady-state exposures and was not associated with a higher number or severity of AEs, implying a high therapeutic index of rezafungin. Overall, no evidence for the adverse effects of rezafungin was found from electrocardiogram or echocardiogram data.

In the phase II evaluation, the randomized, double-blind STRIVE trial (NCT02734862) aimed to evaluate the safety and efficacy of intravenous rezafungin for the treatment of candidemia and invasive candidiasis [185]. The most common TEAEs were mild-to-moderate diarrhea, fever, hypokalemia, and vomiting. No trend was seen between the rezafungin and SOC groups, therefore the STRIVE trial was considered to further validate the robust safety profile of rezafungin at the once-weekly dosing regimen.

A second phase II study, the randomized, multicenter, open-label, sponsor-blinded, active-controlled, dose-ranging RADIANT trial (RADIANT, NCT02733432), was designed to investigate gel and ointment topical formulations of rezafungin for the treatment of acute VVC, with and without a history of recurrence [205]. In three treatment arms, both rezafungin formulations and SOC with oral fluconazole were compared. Most treatment-emergent AEs were unrelated to the study drugs, and all were mild or moderate in intensity. Infections were the most common events across all treatment groups. Vaginal symptoms such as pain and dyspareunia occurred most frequently with the $6 \%$ rezafungin ointment and no serious AEs were reported. Overall, both topical formulations of rezafungin were safe and well tolerated.

For the assessment of subcutaneous route of administration for rezafungin, another double-blind, placebo-controlled phase I study (NCT04117607) was conducted in heathy subjects to determine the safety, tolerability, and pharmacokinetics of subcutaneous application. Despite reported good preclinical tolerability [206], the study was terminated because of the formation of injection-site skin nodules.

3 5.3.2 Efficacy In the phase II efficacy analysis of the STRIVE trial for the treatment of candidemia and invasive candidiasis, the primary efficacy endpoint was overall success at day 14 as demonstrated by mycological eradication and clinical cure [185]. Rezafungin was dosed at either $400 \mathrm{mg}$ weekly or $400 \mathrm{mg}$ for the first week and $200 \mathrm{mg}$ weekly thereafter and compared to SOC with caspofungin once daily and optional switch to oral fluconazole. The rezafungin 400-mg/200-mg regimen showed the greatest overall cure with the highest clinical and mycological cure rates and lowest rate of all-cause mortality at day 30 across all treatment arms. Candidemia cleared more rapidly in the rezafungin-treated patients compared with SOC treatment, possibly reflecting greater fungicidal activity with frontloaded drug exposure, and demonstrated high rates of early treatment efficacy in patients with candidemia [207]. Clinical cure rates were also highest with rezafungin $400 \mathrm{mg} / 200$ $\mathrm{mg}$ when differentiating between $C$. albicans and non-albicans Candida spp. Of note, certain forms of invasive candidiasis such as osteomyelitis, endocarditis or myocarditis, and endophthalmitis were excluded. Apparent differences between the two rezafungin groups raised the discussion of paradoxical growth with higher concentrations. Both in vitro and animal studies of other echinocandins have reported this phenomenon whereby the fungal burden increases at doses above a certain threshold [208]. However, the differences with rezafungin occurred on day 5 , when both treatment arms had received a similar 400-mg dose [185]. A paradoxical growth effect therefore appears unlikely.

Efficacy outcomes of the phase II RADIANT trial were measured as clinical and mycological cures of acute VVC by changes in vaginal scoring system and mycological culture [205]. Two topical formulations of rezafungin were similar in efficacy to each other but lower in clinical and mycological cure rates compared with SOC. Fluconazole also maintained the highest cure rate regardless of infection 
severity or recurrence. Most subjects with non-albicans Candida infections demonstrated treatment failure across all cohorts. Based on these unfavorable results, the development of topical formulation in VVC was discontinued [209]. A non-interventional extension study (NCT02888197) was conducted to follow up on participants who completed the RADIANT trial without recurrence at day 28 visit, although results are not yet available.

3.5.3.3 Ongoing Studies Currently, two phase III trials are recruiting patients to further determine the impact and future of rezafungin for the treatment of invasive candidiasis (ReSTORE, NCT03667690) and for the prevention of IFD (ReSPECT, NCT04368559) (Fig. 3; Table 5 of the ESM). The ReSTORE trial is a multicenter, randomized, doubleblind study examining the rezafungin 400-mg/200-mg once-weekly regimen for the treatment of candidemia and invasive candidiasis. The active comparator is intravenous caspofungin followed by optional fluconazole step-down. The primary endpoint is day 30 all-cause mortality and day 14 global cure measured by clinical, radiological, and mycological indices.

The randomized, double-blind, controlled phase III trial ReSPECT is intended to evaluate rezafungin for the prevention of IFD including Candida spp., Aspergillus spp., and $P$. jirovecii in patients undergoing allogeneic blood and marrow transplantation. Rezafungin $400 \mathrm{mg} / 200 \mathrm{mg}$ once weekly is compared with a standard regimen containing daily azole prophylaxis with fluconazole or posaconazole and anti-Pneumocystis jirovecii prophylaxis with oral trimethoprim-sulfamethoxazole. Fungal-free day 90 survival will be evaluated as the primary outcome.

\subsubsection{Future Role}

The strengths of the echinocandin class combined with the prolonged half-life and robust safety profile of rezafungin open several possibilities for future use. Rezafungin may enable earlier hospital discharge and extended outpatient access to the treatment of invasive candidiasis. Although in vitro susceptibility results are encouraging, further studies are needed to determine the efficacy of rezafungin in the treatment of invasive aspergillosis [192, 199]. Its use as a prophylactic agent for preventing invasive fungal disease caused by Candida spp., Aspergillus spp., and P. jirovecii could overcome current multidrug regimens, and the onceweekly dosing with limited drug-drug interactions may be attractive also for prophylaxis during the early phase after solid organ transplantation (e.g., liver) (Table 1). A frontloaded drug exposure suggests advantages in preventing the selection of resistant strains [210].

\section{Discussion and Conclusions}

There are a number of promising antifungal agents currently in late-stage clinical development. This review highlights the most advanced and promising candidates for timely regulatory approval. These antifungal treatments offer significant advantages in terms of spectrum of activity, tolerability, drug-drug interactions and/or route of administration that have the potential to significantly change the field of clinical mycology as we know it. While current clinical trials often focus on the treatment of refractory, resistant, or breakthrough infections [211], the drugs highlighted in this review-once approved-will likely soon have additional indications and broader clinical use because of the advantages in pharmacokinetics, limited drug-drug interactions, and generally very good tolerability. In addition, three of the drugs discussed in this review have novel mechanisms of action. While these new antifungal drugs are promising, there are still gaps of knowledge regarding their pharmacokinetics and pharmacodynamics, and whether therapeutic drug monitoring may be required. Only once they are used in real-world scenarios, we will find out how prone these new drugs will be to development of novel drug resistance mechanisms. Furthermore, some of the new drugs may have a narrower spectrum of activity compared with some of the currently available broad-spectrum agents and may therefore be less promising for empirical therapy.

In summary, fosmanogepix has a novel mechanism of action and inhibits the fungal enzyme Gwt1. Dosed orally or intravenously, fosmanogepix has a broad spectrum of action against most molds including most of the endemic fungi and most Candida spp., although it lacks activity against $C$. krusei and has variable activity against Mucor and Rhizopus spp. Given its favorable side-effect profile and broad spectrum of activity, fosmanogepix will likely serve as a good treatment option for a broad spectrum of infections. Ibrexafungerp, a first-in-class triterpenoid, is currently available only in oral formulation and is approved by the FDA for the treatment of $\mathrm{VVC}$, although additional approvals are likely forthcoming and it is currently being evaluated to be given intravenously as well. It has good coverage against Aspergillus, most of the endemic fungi, and particularly Candida spp. although activity against other molds including Mucor, Rhizopus, Fusarium, and Scopulariopsis spp. is lacking. Given its oral formulation, ibrexafungerp will likely be a good primary and step-down option for infections from Candida spp., and it may play a role in the treatment of aspergillosis as well. Olorofim is a dihyroorotate dehydrogenase enzyme inhibitor available in oral formulation with good activity against Aspergillus spp., L. prolificans, and Scedosporium spp., and many of the endemic fungi. It appears well tolerated with few drug-drug interactions and 
likely will play a role against difficult-to-treat infections such as those caused by L. prolificans and coccidioidomycoses, which require prolonged treatment courses. Opelconazole is a novel triazole that has been optimized for inhalation and has activity against $C$. neoformans and gattii, and many Aspergillus spp. Opelconazole may have a nice niche for the prophylaxis and treatment of primarily airway-invasive infections where high drug concentrations can be achieved via inhalation without the systemic side effects. Last, rezafungin is a once-weekly intravenous echinocandin with good activity against Aspergillus spp. and Candida spp. as well as $P$. jirovecii. Rezafungin may serve as a good option for prophylaxis after solid organ transplantation or allow for earlier hospital discharge and access to extended outpatient therapy given its once-weekly dosing.

Despite the promising antifungal drugs outlined in this review, there are still remaining unmet needs in the treatment of fungal infections. For example, even with these new options there are still too few antifungal drugs that are well tolerated and with good activity against the Mucorales, with novel treatment options for mucormycosis probably presenting the biggest need currently. In addition, while there are new treatment options against multi-resistant non-Aspergillus spp. including Fusarium spp., Scedosporium spp., and $L$. prolificans, given that these infections can be very difficult to treat and may develop further resistance, more treatment options are likely needed. Last, there are still no antifungal drugs that can eradicate disseminated infection from $\mathrm{Coc}$ cidioides spp. and thus lifelong treatment is still required. Thus, despite the promise that these new antifungal drug options hold, continued research and development into new options including drugs from novel antifungal classes will help replenish the current antifungal armamentarium.

Supplementary Information The online version contains supplementary material available at https://doi.org/10.1007/s40265-021-01611-0.

Acknowledgements We thank Matteo Oliverio, medical illustrator, for creating the figures. We thank Scynexis, Amplyx/Pfizer, Pulmocide, F2G, and Cidara for providing data on the activity of their respective antifungal drugs and details on their clinical trials.

\section{Declarations}

Funding Open access funding provided by Medical University of Graz. MH is supported by the National Institutes of Health (UL1TR001442). No other funding was obtained for this analysis.

Conflicts of interest/competing interests $\mathrm{MH}$ has received research funding from Gilead, Astellas, Pfizer, Merck, Scynexis, F2G, Pulmocide and Amplyx. RS has nothing to disclose. ME has nothing to disclose. AA has nothing to disclose. OAC reports grants or contracts from Amplyx, Basilea, BMBF, Cidara, DZIF, EU-DG RTD (101037867), F2G, Gilead, Matinas, MedPace, MSD, Mundipharma, Octapharma, Pfizer, and Scynexis; consulting fees from Amplyx, Biocon, Biosys, Cidara, Da Volterra, Gilead, Matinas, MedPace, Menarini, Molecular
Partners, MSG-ERC, Noxxon, Octapharma, PSI, Scynexis, and Seres; honoraria for lectures from Abbott, Al-Jazeera Pharmaceuticals, Astellas, Grupo Biotoscana/United Medical/Knight, Hikma, MedScape, MedUpdate, Merck/MSD, Mylan, and Pfizer; payment for expert testimony from Cidara; participation on a Data Safety Monitoring Board or Advisory Board from Actelion, Allecra, Cidara, Entasis, IQVIA, Jannsen, MedPace, Paratek, PSI, and Shionogi; a pending patent currently reviewed at the German Patent and Trade Mark Office; other interests from DGHO, DGI, ECMM, ISHAM, MSG-ERC, and Wiley. RK has nothing to disclose. CLF reports grants, consulting fees, support for travel to meetings and payment for lectures including service on speakers bureaus from Gilead Sciences, Astellas Pharma, Merck Sharp and Dahme, Basilea, and Angelini. JP has nothing to disclose. AS has received grant funding from Astellas and Mayne, and consulting fees from Scynexis and Mayne. GRT has received honoraria from Amplyx, Cidara, Mayne Pharma, Pfizer, and Scynexis. NW has received research funding from Astellas, bioMerieux, Cepheid, Covance, F2G, and Sfunga, and has served on an advisory board for Mayne Pharma. JDJ has received research funding from Astellas, Pfizer, and F2G.

Ethics Approval, Consent to Participate, Consent for Publication, Code Availability Not applicable.

Availability of Data and Material Data available upon request.

Authors contribution $\mathrm{MH}$ had the idea for the review and coordinated the review process. All authors performed the literature search and data analysis for their assigned sections and drafted their assigned sections. $\mathrm{MH}$ and RS created the first draft of the manuscript and developed the Figures and Tables in the review. All authors read, critically revised andapproved the final manuscript.

Open Access This article is licensed under a Creative Commons Attribution-NonCommercial 4.0 International License, which permits any non-commercial use, sharing, adaptation, distribution and reproduction in any medium or format, as long as you give appropriate credit to the original author(s) and the source, provide a link to the Creative Commons licence, and indicate if changes were made. The images or other third party material in this article are included in the article's Creative Commons licence, unless indicated otherwise in a credit line to the material. If material is not included in the article's Creative Commons licence and your intended use is not permitted by statutory regulation or exceeds the permitted use, you will need to obtain permission directly from the copyright holder. To view a copy of this licence, visit http://creativecommons.org/licenses/by-nc/4.0/.

\section{References}

1. Cornely OA, Alastruey-Izquierdo A, Arenz D, Chen SCA, Dannaoui $\mathrm{E}$, Hochhegger $\mathrm{B}$, et al. Global guideline for the diagnosis and management of mucormycosis: an initiative of the European Confederation of Medical Mycology in cooperation with the Mycoses Study Group Education and Research Consortium. Lancet Infect Dis. 2019;19(12):e405-e421.

2. Hoenigl M, Salmanton-García J, Walsh TJ, Nucci M, Neoh CF, Jenks JD, et al. Global guideline for the diagnosis and management of rare mould infections: an initiative of the European Confederation of Medical Mycology in cooperation with the International Society for Human and Animal Mycology and the American Society for Microbiology. Lancet Infect Dis. 2021;21(8):e246-e257. 
3. Salmanton-GarcÍa J, Koehler P, Kindo A, Falces-Romero I, Garć́a-RodrÍguez J, RÁČil Z, et al. Needles in a haystack: extremely rare invasive fungal infections reported in FungiScope ${ }^{\circledR}$ - Global Registry for Emerging Fungal Infections. J Infect. 2020;81(5):802-15.

4. Jenks JD, Seidel D, Cornely OA, Chen S, van Hal S, Kauffman $\mathrm{C}$, et al. Voriconazole plus terbinafine combination antifungal therapy for invasive Lomentospora prolificans infections: analysis of 41 patients from the FungiScope ${ }^{\circledR}$ registry 2008-2019. Clin Microbiol Infect. 2020;S1198-743X(20)30037-9.

5. Arastehfar A, de Almeida Júnior JN, Perlin DS, Ilkit M, Boekhout T, Colombo AL. Multidrug-resistant Trichosporon species: underestimated fungal pathogens posing imminent threats in clinical settings. Crit Rev Microbiol. 2021:1-20.

6. Jenks JD, Gangneux JP, Schwartz IS, Alastruey-Izquierdo A, Lagrou K, Thompson III GR, et al. Diagnosis of breakthrough fungal infections in the clinical mycology laboratory: an ECMM consensus statement. J Fungi. 2020;6(4).

7. Chowdhary A, Prakash A, Sharma C, Kordalewska M, Kumar A, Sarma S, et al. A multicentre study of antifungal susceptibility patterns among 350 Candida auris isolates (2009-17) in India: role of the ERG11 and FKS1 genes in azole and echinocandin resistance. J Antimicrob Chemother. 2018;73:891-9.

8. Schauwvlieghe AFAD, de Jonge N, van Dijk K, Verweij PE, Bruggemann RJ, Biemond BJ, et al. The diagnosis and treatment of invasive aspergillosis in Dutch haematology units facing a rapidly increasing prevalence of azole-resistance: a nationwide survey and rationale for the DB-MSG 002 study protocol. Mycoses. 2018;61:656-64.

9. Alastruey-Izquierdo A, Mellado E, Peláez T, Pemán J, Zapico S, Alvarez M, et al. Population-based survey of filamentous fungi and antifungal resistance in Spain (FILPOP Study). Antimicrob Agents Chemother. 2013;57(7):3380-7.

10. Arastehfar A, Carvalho A, van de Veerdonk FL, Jenks JD, Koehler P, Krause R, et al. COVID-19 associated pulmonary aspergillosis (CAPA): from immunology to treatment. J Fungi. 2020;6(2):91.

11. Prattes J, Wauters J, Giacobbe DR, Lagrou K, Hoenigl M. Diagnosis and treatment of COVID-19 associated pulmonary apergillosis in critically ill patients: results from a European Confederation of Medical Mycology Registry. Intensive Care Med. 2021;1-3. https://doi.org/10.1007/s00134-021-06471-6.

12. Prattes J, Koehler P, Hoenigl M. COVID-19 associated pulmonary aspergillosis: regional variation in incidence and diagnostic challenges. Intensive Care Med. 2021;1-2. https://doi.org/10. 1007/s00134-021-06510-2

13. Prattes J, Wauters J, Giacobbe DR, Salmanton-García J, Maertens J, Bourgeois M, et al. Risk factors and outcome of pulmonary aspergillosis in critically ill coronavirus disease 2019 patients: a multinational observational study by the European Confederation of Medical Mycology. Clin Microbiol Infect. 2021;S1198-743X(21)00474-2.

14. Rudramurthy SM, Hoenigl M, Meis JF, Cornely OA, Muthu V, Gangneux JP, et al. ECMM/ISHAM recommendations for clinical management of COVID-19 associated mucormycosis in lowand middle-income countries. Mycoses. 2021;64(9):1028-37.

15. Hoenigl MS, Carvalho D, Rudramurthy SM, Arastehfar A, Gangneux JP, Nasir N, et al.; ECMM and ISHAM Collaborators. The emergence of COVID-19 associated mucormycosis: analysis of cases from 18 countries. https://ssrn.com/abstract $=3844587$ or https://doi.org/10.2139/ssrn.3844587. Accessed 25 Sep 2021.

16. Koehler P, Bassetti M, Chakrabarti A, Chen SCA, Colombo AL, Hoenigl M, et al. Defining and managing COVID-19-associated pulmonary aspergillosis: the 2020 ECMM/ISHAM consensus criteria for research and clinical guidance. Lancet Infect Dis. 2021;21(6):e149-e162.
17. Jenks JD, Salzer HJ, Prattes J, Krause R, Buchheidt D, Hoenigl $\mathrm{M}$. Spotlight on isavuconazole in the treatment of invasive aspergillosis and mucormycosis: design, development, and place in therapy. Drug Design Dvelop Ther. 2018;12:1033-44.

18. Perlin DS, Rautemaa-Richardson R, Alastruey-Izquierdo A. The global problem of antifungal resistance: prevalence, mechanisms, and management. Lancet Infect Dis. 2017;17(12):e383-92.

19. Berman J, Krysan DJ. Drug resistance and tolerance in fungi. Nat Rev Microbiol. 2020;18(6):319-31.

20. Arastehfar A, Hilmioğlu-Polat S, Daneshnia F, Hafez A, Salehi M, Polat F, et al. Recent increase in the prevalence of fluconazole-non-susceptible Candida tropicalis blood isolates in Turkey: clinical implication of azole-non-susceptible and fluconazole tolerant phenotypes and genotyping. Front Microbiol. 2020;11:587278.

21. Astvad KMT, Sanglard D, Delarze E, Hare RK, Arendrup MC. Implications of the EUCAST trailing phenomenon in Candida tropicalis for the in vivo susceptibility in invertebrate and murine models. Antimicrob Agents Chemother. 2018;62(12):e01624-18.

22. Arastehfar A, Kargar ML, Mohammadi SR, Roudbary M, Ghods $\mathrm{N}$, Haghighi L, et al. A high rate of recurrent vulvovaginal candidiasis and therapeutic failure of azole derivatives among Iranian women. Front Microbiol. 2021;12:655069.

23. Rosenberg A, Ene IV, Bibi M, Zakin S, Segal ES, Ziv N, et al. Antifungal tolerance is a subpopulation effect distinct from resistance and is associated with persistent candidemia. Nat Commun. 2018;9:2470.

24. Sionov E, Chang YC, Garraffo HM, Kwon-Chung KJ. Heteroresistance to fluconazole in Cryptococcus neoformans is intrinsic and associated with virulence. Antimicrob Agents Chemother. 2009;53:2804-15.

25. Todd RT, Selmecki A. Expandable and reversible copy number amplification drives rapid adaptation to antifungal drugs. eLife. 2020;9:e58349.

26. Selmecki A, Forche A, Berman J. Aneuploidy and isochromosome formation in drug-resistant Candida albicans. Science. 2006;313:367-70.

27. Sionov E, Lee H, Chang YC, Kwon-Chung KJ. Cryptococcus neoformans overcomes stress of azole drugs by formation of disomy in specific multiple chromosomes. PLoS Pathog. 2010;6:e1000848.

28. Healey KR, Perlin DS. Fungal resistance to echinocandins and the MDR phenomenon in Candida glabrata. J Fungi. 2018;4(3):105.

29. Arastehfar A, Gabaldón T, Garcia-Rubio R, Jenks JD, Hoenigl $\mathrm{M}$, Salzer HJF, et al. Drug-resistant fungi: an emerging challenge threatening our limited antifungal armamentarium. Antibiotics. 2020;9(12):877.

30. Arastehfar A, Lass-Flörl C, Garcia-Rubio R, Daneshnia F, Ilkit $\mathrm{M}$, Boekhout $\mathrm{T}$, et al. The quiet and underappreciated rise of drug-resistant invasive fungal pathogens. J Fungi. 2020;6(3):138.

31. Arastehfar A, Daneshnia F, Hilmioglu-Polat S, Ilkit M, Yasar M, Polat F, et al. Genetically related micafungin-resistant Candida parapsilosis blood isolates harbouring novel mutation R658G in hotspot 1 of Fks1p: a new challenge? J Antimicrob Chemother. 2021;76:418-22.

32. Arastehfar A, Carvalho A, Houbraken J, Lombardi L, GarciaRubio R, Jenks JD, et al. Aspergillus fumigatus and aspergillosis: from basics to clinics. Stud Mycol. 2021;100:100115.

33. Warrilow AG, Nishimoto AT, Parker JE, Price CL, Flowers SA, Kelly DE, et al. The evolution of azole resistance in Candida albicans sterol 14 $\alpha$-demethylase (CYP51) through incremental amino acid substitutions. Antimicrob Agents Chemother. 2019;63(5):e02586-18.

34. Bosco-Borgeat ME, Mazza M, Taverna CG, Córdoba S, Murisengo OA, Vivot W, et al. Amino acid substitution in 
Cryptococcus neoformans lanosterol 14- $\alpha$-demethylase involved in fluconazole resistance in clinical isolates. Rev Argent Microbiol. 2016;48:137-42.

35. Sionov E, Chang YC, Garraffo HM, Dolan MA, Ghannoum MA, Kwon-Chung KJ. Identification of a Cryptococcus neoformans cytochrome P450 lanosterol 14 $\alpha$-demethylase (Erg 11) residue critical for differential susceptibility between fluconazole/voriconazole and itraconazole/posaconazole. Antimicrob Agents Chemother. 2012;56:1162-9.

36. Arastehfar A, Daneshnia F, Hilmioğlu-Polat S, Fang W, Yaşar M, Polat F, et al. First report of candidemia clonal outbreak caused by emerging fluconazole-resistant Candida parapsilosis isolates harboring Y132F and/or Y132F+K143R in Turkey. Antimicrob Agents Chemother. 2020;64(10):e01001-20.

37. Thomaz DY, de Almeida Jr JN, Sejas ONE, Del Negro GMB, Carvalho GOMH, Gimenes VMF, et al. Environmental clonal spread of azole-resistant Candida parapsilosis with Erg11-Y132F mutation causing a large candidemia outbreak in a Brazilian Cancer Referral Center. J Fungi. 2021.

38. Leonardelli F, Macedo D, Dudiuk C, Cabeza MS, Gamarra S, Garcia-Effron G. Aspergillus fumigatus intrinsic fluconazole resistance is due to the naturally occurring T301I substitution in Cyp51Ap. Antimicrob Agents Chemother. 2016;60:5420-6.

39. Yang H, Tong J, Lee CW, Ha S, Eom SH, Im YJ. Structural mechanism of ergosterol regulation by fungal sterol transcription factor Upc2. Nat Commun. 2015;6:6129.

40. Rybak JM, Muñoz JF, Barker KS, Parker JE, Esquivel BD, Berkow EL, et al. Mutations in TAC1B: a novel genetic determinant of clinical fluconazole resistance in Candida auris. mBio. 2020;11(3):e00365-20.

41. Arastehfar A, Daneshnia F, Hafez A, Khodavaisy S, Najafzadeh M-J, Charsizadeh A, et al. Antifungal susceptibility, genotyping, resistance mechanism, and clinical profile of Candida tropicalis blood isolates. Med Mycol. 2020;58:766-73.

42. Coste AT, Karababa M, Ischer F, Bille J, Sanglard D. TAC1, transcriptional activator of CDR genes, is a new transcription factor involved in the regulation of Candida albicans $\mathrm{ABC}$ transporters CDR1 and CDR2. Eukaryot Cell. 2004;3:1639-52.

43. Gsaller F, Hortschansky P, Furukawa T, Carr PD, Rash B, Capilla $\mathrm{J}$, et al. Sterol biosynthesis and azole tolerance is governed by the opposing actions of SrbA and the CCAAT binding complex. PLoS Pathog. 2016;12:e1005775.

44. Sanguinetti M, Posteraro B, La Sorda M, Torelli R, Fiori B, Santangelo R, et al. Role of AFR1, an ABC transporter-encoding gene, in the in vivo response to fluconazole and virulence of Cryptococcus neoformans. Infect Immun. 2006;74:1352-9.

45. Basso LRJ, Gast CE, Bruzual I, Wong B. Identification and properties of plasma membrane azole efflux pumps from the pathogenic fungi Cryptococcus gattii and Cryptococcus neoformans. J Antimicrob Chemother. 2015;70:1396-407.

46. Liu Z, Rossi JM, Myers LC. Candida albicans Zn cluster transcription factors Tac1 and Znc1 are activated by farnesol to upregulate a transcriptional program including the multidrug efflux pump CDR1. Antimicrob Agents Chemother. 2018;62(11):e00968-18.

47. Ferrari S, Sanguinetti M, Torelli R, Posteraro B, Sanglard D. Contribution of CgPDR1-regulated genes in enhanced virulence of azole-resistant Candida glabrata. PLoS ONE. 2011;6:e17589.

48. Vale-Silva L, Ischer F, Leibundgut-Landmann S, Sanglard D. Gain-of-function mutations in PDR1, a regulator of antifungal drug resistance in Candida glabrata, control adherence to host cells. Infect Immun. 2013;81:1709-20.

49. Paul S, Stamnes M, Thomas GH, Liu H, Hagiwara D, Gomi $\mathrm{K}$, et al. AtrR is an essential determinant of azole resistance in Aspergillus fumigatus. mBio. 2019;10(2):e02563-18.
50. Hagiwara D, Miura D, Shimizu K, Paul S, Ohba A, Gonoi T, et al. A novel Zn2-Cys6 transcription factor AtrR plays a key role in an azole resistance mechanism of Aspergillus fumigatus by co-regulating cyp51A and cdr1B expressions. PLoS Pathog. 2017;13:e1006096.

51. Ukai Y, Kuroiwa M, Kurihara N, Naruse H, Homma T, Maki $\mathrm{H}$, et al. Contributions of yap1 mutation and subsequent atrF upregulation to voriconazole resistance in Aspergillus flavus. Antimicrob Agents Chemother. 2018;62(11):e01216-18.

52. Vale-Silva LA, Moeckli B, Torelli R, Posteraro B, Sanguinetti M, Sanglard D. Upregulation of the adhesin gene EPA1 mediated by PDR1 in Candida glabrata leads to enhanced host colonization. mSphere. 2016;1(2):e00065-15.

53. Vale-Silva L, Ischer F, Leibundgut-Landmann S, Sanglard D. Gain-of-function mutations in PDR1, a regulator of antifungal drug resistance in Candida glabrata, control adherence to host cells. Infect Immun. 2013;81(5):1709-20.

54. Chung D, Barker BM, Carey CC, Merriman B, Werner ER, Lechner BE, et al. ChIP-seq and in vivo transcriptome analyses of the Aspergillus fumigatus SREBP SrbA reveals a new regulator of the fungal hypoxia response and virulence. PLoS Pathog. 2014;10(11):e1004487.

55. Hagiwara D, Miura D, Shimizu K, Paul S, Ohba A, Gonoi T, et al. A novel Zn2-Cys6 transcription factor AtrR plays a key role in an azole resistance mechanism of Aspergillus fumigatus by co-regulating cyp51A and cdr1B expressions. PLoS Pathog. 2017;13(1):e1006096.

56. Willger SD, Puttikamonkul S, Kim KH, Burritt JB, Grahl N, Metzler LJ, et al. A sterol-regulatory element binding protein is required for cell polarity, hypoxia adaptation, azole drug resistance, and virulence in Aspergillus fumigatus. PLoS Pathog. 2008;4(11):e1000200.

57. Jiménez-Ortigosa C, Moore C, Denning DW, Perlin DS. Emergence of echinocandin resistance due to a point mutation in the fks 1 gene of Aspergillus fumigatus in a patient with chronic pulmonary aspergillosis. Antimicrob Agents Chemother. 2017;61(12):e01277-17.

58. Khan Z, Ahmad S, Mokaddas E, Meis JF, Joseph L, Abdullah A, et al. Development of Echinocandin resistance in Candida tropicalis following short-term exposure to caspofungin for empiric therapy. Antimicrob Agents Chemother. 2018;62(4):e01926-17.

59. Lackner M, Tscherner M, Schaller M, Kuchler K, Mair C, Sartori $\mathrm{B}$, et al. Positions and numbers of FKS mutations in Candida albicans selectively influence in vitro and in vivo susceptibilities to echinocandin treatment. Antimicrob Agents Chemother. 2014;58:3626-35.

60. Kordalewska M, Lee A, Park S, Berrio I, Chowdhary A, Zhao $\mathrm{Y}$, et al. Understanding echinocandin resistance in the emerging pathogen Candida auris. Antimicrob Agents Chemother. 2018;62(6):e00238-18.

61. Pham CD, Iqbal N, Bolden CB, Kuykendall RJ, Harrison LH, Farley MM, et al. Role of FKS mutations in Candida glabrata: MIC values, echinocandin resistance, and multidrug resistance. Antimicrob Agents Chemother. 2014;58:4690-6.

62. Arastehfar A, Daneshnia F, Salehi M, Yaşar M, Hoşbul T, Ilkit $\mathrm{M}$, et al. Low level of antifungal resistance of Candida glabrata blood isolates in Turkey: fluconazole minimum inhibitory concentration and FKS mutations can predict therapeutic failure. Mycoses. 2020;63(9):911-20.

63. Satish S, Jiménez-Ortigosa C, Zhao Y, Lee MH, Dolgov E, Krüger T, et al. Stress-induced changes in the lipid microenvironment of $\beta$-(1,3)-d-glucan synthase cause clinically important echinocandin resistance in Aspergillus fumigatus. mBio. 2019;10(3):e00779-19. 
64. Shaw KJ, Ibrahim AS. Fosmanogepix: a review of the first-inclass broad spectrum agent for the treatment of invasive fungal infections. J Fungi. 2020;6(4):239.

65. Miyazaki M, Horii T, Hata K, Watanabe NA, Nakamoto K, Tanaka K, et al. In vitro activity of E1210, a novel antifungal, against clinically important yeasts and molds. Antimicrob Agents Chemother. 2011;55(10):4652-8.

66. Tsukahara K, Hata K, Nakamoto K, Sagane K, Watanabe NA, Kuromitsu J, et al. Medicinal genetics approach towards identifying the molecular target of a novel inhibitor of fungal cell wall assembly. Mol Microbiol. 2003;48(4):1029-42.

67. Chaffin WL. Candida albicans cell wall proteins. Microbiol Mol Biol Rev. 2008;72(3):495-544.

68. Fu Y, Luo G, Spellberg BJ, Edwards JE Jr, Ibrahim AS. Gene overexpression/suppression analysis of candidate virulence factors of Candida albicans. Eukaryot Cell. 2008;7(3):483-92.

69. Hoyer LL. The ALS gene family of Candida albicans. Trends Microbiol. 2001;9(4):176-80.

70. Kapteyn JC, Hoyer LL, Hecht JE, Muller WH, Andel A, Verkleij AJ, et al. The cell wall architecture of Candida albicans wild-type cells and cell wall-defective mutants. Mol Microbiol. 2000;35(3):601-11.

71. Sheppard DC, Yeaman MR, Welch WH, Phan QT, Fu Y, Ibrahim AS, et al. Functional and structural diversity in the Als protein family of Candida albicans. J Biol Chem. 2004;279(29):30480-9.

72. Watanabe NA, Miyazaki M, Horii T, Sagane K, Tsukahara K, Hata K. E1210, a new broad-spectrum antifungal, suppresses Candida albicans hyphal growth through inhibition of glycosylphosphatidylinositol biosynthesis. Antimicrob Agents Chemother. 2012;56(2):960-71.

73. Zhao M, Lepak AJ, VanScoy B, Bader JC, Marchillo K, Vanhecker J, et al. In vivo pharmacokinetics and pharmacodynamics of APX001 against Candida spp. in a neutropenic disseminated candidiasis mouse model. Antimicrob Agents Chemother. 2018;62(4):e02542-17.

74. Zhao Y, Lee MH, Paderu P, Lee A, Jimenez-Ortigosa C, Park S, et al. Significantly Improved pharmacokinetics enhances in vivo efficacy of APX001 against echinocandin- and multidrug-resistant Candida isolates in a mouse model of invasive candidiasis. Antimicrob Agents Chemother. 2018;62(10):e00425-18.

75. Wiederhold NP, Najvar LK, Shaw KJ, Jaramillo R, Patterson H, Olivo M, et al. Efficacy of delayed therapy with Fosmanogepix (APX001) in a murine model of Candida auris invasive candidiasis. Antimicrob Agents Chemother. 2019;63(11):e01120-19.

76. Zhao M, Lepak AJ, Marchillo K, Vanhecker J, Sanchez H, Ambrose PG, et al. APX001 pharmacokinetic/pharmacodynamic target determination against Aspergillus fumigatus in an in vivo model of invasive pulmonary aspergillosis. Antimicrob Agents Chemother. 2019;63(4).

77. Pfaller MA, Hata K, Jones RN, Messer SA, Moet GJ, Castanheira M. In vitro activity of a novel broad-spectrum antifungal, E1210, tested against Candida spp. as determined by CLSI broth microdilution method. Diagn Microbiol Infect Dis. 2011;71(2):167-70.

78. Castanheira M, Duncanson FP, Diekema DJ, Guarro J, Jones RN, Pfaller MA. Activities of E1210 and comparator agents tested by CLSI and EUCAST broth microdilution methods against Fusarium and Scedosporium species identified using molecular methods. Antimicrob Agents Chemother. 2012;56(1):352-7.

79. Pfaller MA, Duncanson F, Messer SA, Moet GJ, Jones RN, Castanheira M. In vitro activity of a novel broad-spectrum antifungal, E1210, tested against Aspergillus spp. determined by CLSI and EUCAST broth microdilution methods. Antimicrob Agents Chemother. 2011;55(11):5155-8.

80. Pfaller MA, Watanabe N, Castanheira M, Messer SA, Jones RN. Pre-clinical development of antifungal susceptibility test methods for the testing of the novel antifungal agent E1210 versus Candida: comparison of CLSI and European Committee on Antimicrobial Susceptibility Testing methods. J Antimicrob Chemother. 2011;66(11):2581-4.

81. Gebremariam T, Alkhazraji S, Alqarihi A, Jeon HH, Gu Y, Kapoor M, et al. APX001 is effective in the treatment of murine invasive pulmonary aspergillosis. Antimicrob Agents Chemother. 2019;63(2):e01713-18.

82. Viriyakosol S, Kapoor M, Okamoto S, Covel J, Soltow QA, Trzoss M, et al. APX001 and other Gwt1 inhibitor prodrugs are effective in experimental Coccidioides immitis pneumonia. Antimicrob Agents Chemother. 2019;63(2):e01715-18.

83. Wiederhold NP, Najvar LK, Fothergill AW, McCarthy DI, Bocanegra R, Olivo M, et al. The investigational agent E1210 is effective in treatment of experimental invasive candidiasis caused by resistant Candida albicans. Antimicrob Agents Chemother. 2015;59(1):690-2.

84. Shaw KJ, Schell WA, Covel J, Duboc G, Giamberardino C, Kapoor M, et al. In vitro and in vivo evaluation of APX001A/ APX001 and other Gwt1 inhibitors against Cryptococcus. Antimicrob Agents Chemother. 2018;62(8):e00523-18.

85. Zhu Y, Kilburn S, Kapoor M, Chaturvedi S, Shaw KJ, Chaturvedi $\mathrm{V}$. In vitro activity of Manogepix against multidrug-resistant and panresistant Candida auris from the New York outbreak. Antimicrob Agents Chemother. 2020;64(11):e01124-20.

86. Hager CL, Larkin EL, Long L, Zohra Abidi F, Shaw KJ, Ghannoum MA. In vitro and in vivo evaluation of the antifungal activity of APX001A/APX001 against Candida auris. Antimicrob Agents Chemother. 2018;62(3):e02319-17.

87. Badali H, Patterson HP, Sanders CJ, Mermella B, Gibas CFC, Ibrahim AS, et al. Manogepix, the active moiety of the investigational agent fosmanogepix, demonstrates in vitro activity against members of the Fusarium oxysporum and Fusarium solani species complexes. Antimicrob Agents Chemother. 2021;65(6):e02343-20.

88. Pfaller MA, Huband MD, Flamm RK, Bien PA, Castanheira M. Antimicrobial activity of first-in-class antifungal, manogepix, and comparator agents testing against contemporary invasive fungal isolates from an international surveillance program (20182019): in vitro activity of manogepix against fungal isolates. J Glob Antimicrob Resist. 2021;26:117-27.

89. Rivero-Menendez O, Cuenca-Estrella M, Alastruey-Izquierdo A. In vitro activity of APX001A against rare moulds using EUCAST and CLSI methodologies. J Antimicrob Chemother. 2019;74(5):1295-9.

90. Alkhazraji S, Gebremariam T, Alqarihi A, Gu Y, Mamouei Z, Singh S, et al. Fosmanogepix (APX001) is effective in the treatment of immunocompromised mice infected with invasive pulmonary scedosporiosis or disseminated fusariosis. Antimicrob Agents Chemother. 2020;64(3):e01735-19.

91. Arendrup MC, Chowdhary A, Jorgensen KM, Meletiadis J. Manogepix (APX001A) in vitro activity against Candida auris: head-to-head comparison of EUCAST and CLSI MICs. Antimicrob Agents Chemother. 2020;64(10)64(10):e00656-20.

92. Jorgensen KM, Astvad KMT, Arendrup MC. In vitro activity of manogepix (APX001A) and comparators against contemporary molds: MEC comparison and preliminary experience with colorimetric MIC determination. Antimicrob Agents Chemother. 2020;64(8):e00730-20.

93. Arendrup MC, Jorgensen KM. Manogepix (APX001A) displays potent in vitro activity against human pathogenic yeast, but with an unexpected correlation to fluconazole MICs. Antimicrob Agents Chemother. 2020;64(7):e00429-20

94. Arendrup MC, Chowdhary A, Astvad KMT, Jorgensen KM. APX001A in vitro activity against contemporary blood isolates 
and Candida auris determined by the EUCAST reference method. Antimicrob Agents Chemother. 2018;62(10):e01225-18.

95. Pfaller MA, Huband MD, Flamm RK, Bien PA, Castanheira M. In vitro activity of APX001A (manogepix) and comparator agents against 1,706 fungal isolates collected during an international surveillance program in 2017. Antimicrob Agents Chemother. 2019;63(8):e00840-19.

96. Gebremariam T, Alkhazraji S, Alqarihi A, Wiederhold NP, Shaw KJ, Patterson TF, et al. APX001A protects immunosuppressed mice from Rhizopus delemar infection. Open Forum Infect Dis. 2017;4(Suppl1):S475-S

97. Gebremariam T, Alkhazraji S, Gu Y, Alqarihi A, Mamouei Z, Shaw KJ, et al. 726. APX001 (fosmanogepix) is effective in an immunosuppressed mouse model of Rhizopus oryzae infection. Open Forum Infect Dis. 2019;6(Suppl. 2):S325-6.

98. Gebremariam T, Alkhazraji S, Alqarihi A, Wiederhold NP, Shaw KJ, Patterson TF, et al. Fosmanogepix (APX001) is effective in the treatment of pulmonary murine mucormycosis due to Rhizopus arrhizus. Antimicrob Agents Chemother. 2020;64(6):e00178-20.

99. Ibrahim AG, Gu T, Alkhazraji S, Youssef E, Shaw K. Combination treatment of fosmanogepix and liposomal amphotericin B in treating experimental invasive pulmonary aspergillosis and mucormycosis. 31st ECCMID; 2021; Vienna.

100. Arikan S, Lozano-Chiu M, Paetznick V, Rex JH. In vitro susceptibility testing methods for caspofungin against Aspergillus and Fusarium isolates. Antimicrob Agents Chemother. 2001;45(1):327-30.

101. Hata K, Horii T, Miyazaki M, Watanabe NA, Okubo M, Sonoda $\mathrm{J}$, et al. Efficacy of oral E1210, a new broad-spectrum antifungal with a novel mechanism of action, in murine models of candidiasis, aspergillosis, and fusariosis. Antimicrob Agents Chemother. 2011;55(10):4543-51.

102. Hodges MR, Ople E, Shaw KJ, Mansbach R, Van Marle SJ, Van Hoogdalem E, et al. First-in-human study to assess safety, tolerability and pharmacokinetics of APX001 administered by intravenous infusion to healthy subjects. Open Forum Infect Dis. 2017;4(Suppl_1):S526. https://doi.org/10.1093/ofid/ofx163. 1370.

103. Hodges MR, Ople E, Shaw KJ, Mansbach R, Van Marle SJ, Van Hoogdalem E, et al. Phase 1 study to assess safety, tolerability and pharmacokinetics of single and multiple oral doses of APX001 and to investigate the effect of food on APX001 bioavailability. Open Forum Infect Dis. 2017;4(Suppl_1):S534. https://doi.org/10.1093/ofid/ofx163.1390.

104. Pappas PG, Kullberg BJ, Vazquez JA, Oren I, Rahav G, Aoun M, et al. Clinical safety and efficacy of novel antifungal, Fosmanogepix, in the treatment of candidemia: results from a phase 2 proof of concept trial. IDWeek 2020. In: Programs and Abstracts of the 55th Annual Infectious Diseases Society of America (IDSA) Meeting; October 2020; Philadelphia (PA).

105. Bulpa P, Rahav G, Oren I, Aoun M, Thompson 3rd GR, Pappas PG, et al. Clinical safety and efficacy of fosmanogepix, a novel first-in-class antifungal, in patients with renal insufficiency: subset analysis from a phase 2 candidemia trial. IDWeek 2020. Programs and Abstracts of the 55th Annual Infectious Diseases Society of America (IDSA) Meeting; October 2020; Philadelphia (PA): poster \#1157.

106. Kullberg BB, Pappas L, Vazquez PG, Goosen JA, Arauz J, Paruk $A B$, et al. Clinical efficacy and safety of the novel antifungal fosmanogepix in patients with candidaemia and/or invasive candidiasis caused by Candida auris: results from a phase II proof of concept trial. 31st ECCMID; 2021; Vienna.

107. Bowman JC, Hicks PS, Kurtz MB, Rosen H, Schmatz DM, Liberator PA, et al. The antifungal echinocandin caspofungin acetate kills growing cells of Aspergillus fumigatus in vitro. Antimicrob Agents Chemother. 2002;46(9):3001-12.

108. Ghannoum M, Long L, Larkin EL, Isham N, Sherif R, BorrotoEsoda K, et al. Evaluation of the antifungal activity of the novel oral glucan synthaseiInhibitor SCY-078, singly and in combination, for the treatment of invasive aspergillosis. Antimicrob Agents Chemother. 2018;62(6):e00244-18.

109. Pfaller MA, Messer SA, Motyl MR, Jones RN, Castanheira M. In vitro activity of a new oral glucan synthase inhibitor (MK3118) tested against Aspergillus spp. by CLSI and EUCAST broth microdilution methods. Antimicrob Agents Chemother. 2013;57(2):1065-8.

110. Walker SS, Xu Y, Triantafyllou I, Waldman MF, Mendrick C, Brown N, et al. Discovery of a novel class of orally active antifungal beta-1,3-D-glucan synthase inhibitors. Antimicrob Agents Chemother. 2011;55(11):5099-106.

111. Jiménez-Ortigosa C, Perez WB, Angulo D, Borroto-Esoda K, Perlin DS. De novo acquisition of resistance to SCY-078 in Candida glabrata involves FKS mutations that both overlap and are distinct from those conferring echinocandin resistance. Antimicrob Agents Chemother. 2017;61(9):e00833-17.

112. Nunnally NS, Etienne KA, Angulo D, Lockhart SR, Berkow EL. In vitro activity of Ibrexafungerp, a novel glucan synthase inhibitor against Candida glabrata isolates with FKS mutations. Antimicrob Agents Chemother. 2019;63(11):e01692-19.

113. Wring SA, Randolph R, Park S, Abruzzo G, Chen Q, Flattery A, et al. Preclinical pharmacokinetics and pharmacodynamic target of SCY-078, a first-in-class orally active antifungal glucan synthesis inhibitor, in murine models of disseminated candidiasis. Antimicrob Agents Chemother. 2017;61(4):e02068-16.

114. Gamal A, Chu S, McCormick TS, Borroto-Esoda K, Angulo D, Ghannoum MA. Ibrexafungerp, a novel oral triterpenoid antifungal in development: overview of antifungal activity against Candida glabrata. Front Cell Infect Microbiol. 2021;11:642358.

115. Davis MR, Donnelley MA, Thompson GR. Ibrexafungerp: a novel oral glucan synthase inhibitor. Med Mycol. 2020;58(5):579-92.

116. Wring S, Borroto-Esoda K, Solon E, Angulo D. SCY-078, a novel fungicidal agent, demonstrates distribution to tissues associated with fungal infections during mass balance studies with intravenous and oral [(14)C]SCY-078 in albino and pigmented rats. Antimicrob Agents Chemother. 2019;63(2):e02119-18.

117. Rauseo AM, Coler-Reilly A, Larson L, Spec A. Hope on the horizon: novel fungal treatments in development. Open Forum Infect Dis. 2020;7(2):ofaa016.

118. Larkin EL, Long L, Isham N, Borroto-Esoda K, Barat S, Angulo $\mathrm{D}$, et al. A novel 1,3-beta-d-glucan inhibitor, Ibrexafungerp (formerly SCY-078), shows potent activity in the lower $\mathrm{pH}$ environment of vulvovaginitis. Antimicrob Agents Chemother. 2019;63(5):e02611-18.

119. Wring S, Murphy G, Atiee G, Corr C, Hyman M, Willett M, et al. Clinical pharmacokinetics and drug-drug interaction potential for coadministered SCY-078, an oral fungicidal glucan synthase inhibitor, and tacrolimus. Clin Pharmacol Drug Dev. 2019;8(1):60-9.

120. Ghannoum M, Long L, Larkin EL, Isham N, Sherif R, BorrotoEsoda K, et al. SCY-078, a novel oral glucan synthase inhibitor, for the treatment of invasive aspergillosis: evaluation of antifungal activity singly and in combination. Antimicrob Agents Chemother. 2018;62(6):e00244-18.

121. Cushion M AA, Borroto-Esoda K, Barat S, Angulo D, editor. SCY-078 demonstrates antifungal activity against Pneumocystis in a prophylactic murine model of Pneumocystis pneumonia. ECCMID; 2018; Madrid. 
122. Barat SAB-E K, Angulo D, Ashbaugh A, Cushion M, editor Efficacy of ibrexafungerp (formerly SCY-078) in a murine treatment model of Pneumocystis pneumonia. ASM Microbe. 2019.

123. Schell WA, Jones AM, Borroto-Esoda K, Alexander BD. Antifungal activity of SCY-078 and standard antifungal agents against 178 clinical isolates of resistant and susceptible Candida species. Antimicrob Agents Chemother. 2017;61(11):e01102-17.

124. Ghannoum M, Long L, Isham N, Hager C, Wilson R, Borroto-Esoda $\mathrm{K}$, et al. Activity of a novel 1,3-beta-D-glucan synthase inhibitor, ibrexafungerp (formerly SCY-078), against Candida glabrata. Antimicrob Agents Chemother. 2019;63(12):e01510-19.

125. Zhu YC, Barat SA, Borroto-Esoda K, Angulo D, Chaturvedi S, Chaturvedi V. Pan-resistant Candida auris isolates from the outbreak in New York are susceptible to ibrexafungerp (a glucan synthase inhibitor). Int J Antimicrob Agents. 2020;55(4):105922.

126. Arendrup MC, Jørgensen KM, Hare RK, Chowdhary A. In vitro activity of ibrexafungerp (SCY-078) against Candida auris isolates as determined by EUCAST methodology and comparison with activity against $\mathrm{C}$. albicans and C. glabrata and with the activities of six comparator agents. Antimicrob Agents Chemother. 2020;64(3):e02136-19.

127. Jiménez-Ortigosa C, Paderu P, Motyl MR, Perlin DS. Enfumafungin derivative MK-3118 shows increased in vitro potency against clinical echinocandin-resistant Candida species and Aspergillus species isolates. Antimicrob Agents Chemother. 2014;58(2):1248-51.

128. Marcos-Zambrano LJ, Gomez-Perosanz M, Escribano P, Bouza E, Guinea J. The novel oral glucan synthase inhibitor SCY-078 shows in vitro activity against sessile and planktonic Candida spp. J Antimicrob Chemother. 2017;72(7):1969-76.

129. Pfaller MA, Messer SA, Rhomberg PR, Borroto-Esoda K, Castanheira M. Differential activity of the oral glucan synthase inhibitor SCY-078 against wild-type and echinocandin-resistant strains of Candida species. Antimicrob Agents Chemother. 2017;61(8):e00161-17.

130. Rivero-Menendez O, Soto-Debran JC, Cuenca-Estrella M, Alastruey-Izquierdo A. In vitro activity of Ibrexafungerp against a collection of clinical isolates of Aspergillus, including cryptic species and Cyp51A mutants, using EUCAST and CLSI methodologies. J Fungi. 2021;7(3):232.

131. Lamoth F, Alexander BD. Antifungal activities of SCY-078 (MK-3118) and standard antifungal agents against clinical non-Aspergillus mold isolates. Antimicrob Agents Chemother. 2015;59(7):4308-11.

132. Ghannoum MLL, Sherif R, Abidi FZ, Borroto-Esoda K, Barat $\mathrm{S}$, Angulo D, et al., editors Determination of antifungal activity of SCY-078, a novel glucan synthase inhibitor, against a broad panel of rare pathogenic fungi. ASM Microbe. 2020.

133. Trucksis M, Garrett G, Heriman I. A phase I multiple rising dose study evaluating safety, tolerability, and pharmacokinetics of MK-3118, oral glucan synthase inhibitor, in healthy volunteers. 51st Interscience Conference on Antimicrobial Agents and Chemotherapy (ICAAC); 2011; Chicago (IL).

134. Alexander BD, Cornely O, Pappas P, Miller R, Vazquez JA, Ostrosky-Zeichner L, et al. 1248. Efficacy and safety of oral Ibrexafungerp in 41 patients with refractory fungal diseases, interim analysis of a phase 3 open-label study (FURI). Open Forum Infect Dis. 2020;7(Suppl_1):S642-S.

135. Spec A, Pullman J, Thompson GR, Powderly WG, Tobin EH, Vazquez J, et al. MSG-10: a phase 2 study of oral ibrexafungerp (SCY-078) following initial echinocandin therapy in non-neutropenic patients with invasive candidiasis. J Antimicrob Chemother. 2019;74(10):3056-62.

136. Angulo D, Tufa M, Azie N. A phase $2 b$, dose-selection study evaluating the efficacy and safety of oral ibrexafungerp vs fluconazole in vulvovaginal candidiasis (DOVE). Am J Obstet Gynecol. 2019;221(6):673.

137. Hoenigl M, Cornely OA, Koehler P, Pappas PG, McCarthy T, Miller R, Vazquez J, Sanders JW, Morse CG, et al. Outcomes of oral ibrexafungerp in 33 patients with refractory fungal diseases, interim analysis of a phase 3 open-label study (FURI). 31st ECCMID; 2021; Vienna.

138. Murphy G, Darpo B, Marbury T. Lack of an effect of SCY-078 a novel antifungal agent on QTc interval in healthy subjects. ASM Microbe; 2017; New Orleans (LA).

139. Carruthers C, Barat S, Thomas P, Lewis E. SCY-078, a novel IV/ oral triterpenoid anti-fungal treatment in development for vulvovaginal candidiasis and other severe invasive fungal infections, is not embryo/feto-toxic. Am J Obstet Gynecol. 2018;219(6):646-7.

140. Koehler P, Cornely OA, Angulo D. Favourable clinical outcome of two patients with Candida spp spondylodiscitis treated with oral Ibrexafungerp (formerly SCY-078) from the FURI Study. 29th ECCMID; 2019; Amsterdam.

141. Astvad KMT, Johansen HK, Roder BL, Rosenvinge FS, Knudsen JD, Lemming L, et al. Update from a 12-year nationwide Fungemia surveillance: increasing intrinsic and acquired resistance causes Concern. J Clin Microbiol. 2018;56(4):e01564-17.

142. Azie NJ, D; Ross, C; Breedt, J; Siebert, RS; Angulo, DA. Outcomes of oral ibrexafungerp in the treatment of ten patients with Candida auris infections, from the CARES Study. 31st ECCMID; 2021; Vienna.

143. Helou S, Angulo D. A multicenter, randomized, evaluator blinded, active-controlled study to evaluate the safety and efficacy of oral SCY-078 vs. oral fluconazole in 96 subjects with moderate to severe vulvovaginal candidiasis. Am J Obstet Gynecol. 2017;217(6):720-1.

144. Schwebke JR, Sorkin-Wells V, Azie N, Angulo D, Sobel J. Oral ibrexafungerp efficacy and safety in the treatment of vulvovaginal candidiasis: a phase 3 , randomized, blinded, study vs. placebo (VANISH-303). Am J Obstet Gynecol. 2020;223(6):964-5.

145. US Food and Drug Administration CfDEaR. NDA Multi-disciplinary Review and Evaluation: NDA 214900; BREXAFEMME (ibrexafungerp) 2020. https://www.accessdata.fda.gov/drugs atfda_docs/nda/2021/214900Orig1s000MultidisciplineR.pdf. Accessed 25 Sep 2021.

146. Petraitis V, Petraitiene R, Katragkou A, Maung BBW, Naing E, Kavaliauskas $\mathrm{P}$, et al. Combination therapy with ibrexafungerp (formerly SCY-078), a first-in-class triterpenoid inhibitor of (1-->3)-beta-d-glucan synthesis, and isavuconazole for treatment of experimental invasive pulmonary aspergillosis. Antimicrob Agents Chemother. 2020;64(6):e02429-19.

147. Hoenigl MBA, Gangneux JP, Richardson R, Arikan-Akdagli S, Salmanton-Garcia J, Lagrou K, et al. Outcomes of invasive Candida infections in Europe: results from ECMM Candida III: a multinational European study. 31st ECCMID; 2021; Vienna.

148. Maertens JA, Raad II, Marr KA, Patterson TF, Kontoyiannis DP, Cornely OA, et al. Isavuconazole versus voriconazole for primary treatment of invasive mould disease caused by Aspergillus and other filamentous fungi (SECURE): a phase 3, randomisedcontrolled, non-inferiority trial. Lancet. 2016;387(10020):760-9.

149. Negri CE, Johnson A, McEntee L, Box H, Whalley S, Schwartz JA, et al. Pharmacodynamics of the novel antifungal agent F901318 for acute sinopulmonary aspergillosis caused by Aspergillus flavus. J Infect Dis. 2018;217(7):1118-27.

150. Kennedy T GA, Steiner J. An open-label study in healthy volunteers to evaluate the potential for cytochrome P450 3A4 inhibition by F901318 using oral midazolam as a probe. 27th ECCMID; 2017; Vienna.

151. Kirchhoff L, Dittmer S, Buer J, Rath PM, Steinmann J. In vitro activity of olorofim (F901318) against fungi of the genus, Scedosporium and Rasamsonia as well as against Lomentospora 
prolificans, Exophiala dermatitidis and azole-resistant Aspergillus fumigatus. Int J Antimicrob Agents. 2020;56(3):106105.

152. Georgacopoulos O, Nunnally NS, Ransom EM, Law D, Birch M, Lockhart SR, et al. In vitro activity of novel antifungal olorofim against filamentous fungi and comparison to eight other antifungal agents. J Fungi. 2021;7(5):378.

153. Singh A, Singh P, Meis JF, Chowdhary A. In vitro activity of the novel antifungal olorofim against dermatophytes and opportunistic moulds including Penicillium and Talaromyces species. J Antimicrob Chemother. 2021;76(5):1229-33.

154. Oliver JD, Sibley GEM, Beckmann N, Dobb KS, Slater MJ, McEntee L, et al. F901318 represents a novel class of antifungal drug that inhibits dihydroorotate dehydrogenase. Proc Natl Acad Sci U S A. 2016;113(45):12809-14.

155. Wiederhold NP. Review of the novel investigational antifungal olorofim. J Fungi. 2020;6(3):122.

156. Fothergill AWN, Sibley G, Kennedy A, Oliver J, Birch M, Law D, editors. Spectrum of activity of F901318, the first agent from the new orotomide class of antifungals. ICAAC; 2015; San Diego (CA).

157. Wiederhold NP, Najvar LK, Jaramillo R, Olivo M, Birch M, Law $\mathrm{D}$, et al. The orotomide olorofim Is efficacious in an experimental model of central nervous system coccidioidomycosis. Antimicrob Agents Chemother. 2018;62(9):e00999-18.

158. Zhang J, Liu H, Xi L, Chang YC, Kwon-Chung KJ, Seyedmousavi S. Antifungal susceptibility profiles of olorofim (formerly F901318) and currently available systemic antifungals against mold and yeast phases of Talaromyces marneffei. Antimicrob Agents Chemother. 2021;65(6):e00256-21.

159. Lim W, Eadie K, Konings M, Rijnders B, Fahal AH, Oliver JD, et al. Madurella mycetomatis, the main causative agent of eumycetoma, is highly susceptible to olorofim. J Antimicrob Chemother. 2020;75(4):936-41.

160. Astvad KMT, Jørgensen KM, Hare RK, Datcu R, Arendrup MC. Olorofim susceptibility testing of 1,423 Danish mold isolates obtained in 2018-2019 confirms uniform and broad-spectrum activity. Antimicrob Agents Chemother. 2020;65(1):e01527-20.

161. Rivero-Menendez O, Cuenca-Estrella M, Alastruey-Izquierdo A. In vitro activity of olorofim against clinical isolates of Scedosporium species and Lomentospora prolificans using EUCAST and CLSI methodologies. J Antimicrob Chemother. 2020;75(12):3582-5.

162. Biswas C, Law D, Birch M, Halliday C, Sorrell TC, Rex J, et al. In vitro activity of the novel antifungal compound F901318 against Australian Scedosporium and Lomentospora fungi. Med Mycol. 2018;56(8):1050-4.

163. Wiederhold NP, Law D, Birch M. Dihydroorotate dehydrogenase inhibitor F901318 has potent in vitro activity against Scedosporium species and Lomentospora prolificans. J Antimicrob Chemother. 2017;72(7):1977-80.

164. Müller C, Fietz C, Koehler P, Sibley G, Nforbugwe ACA, Streichert $\mathrm{T}$, et al. Reliable and easy-to-use liquid chromatographytandem mass spectrometry assay for quantification of olorofim (F901318), a novel antifungal drug, in human plasma and serum. Antimicrob Agents Chemother. 2018;62(11):e01356-18.

165. Su H, Zhu M, Tsui CK, van der Lee H, Tehupeiory-Kooreman M, Zoll J, et al. Potency of olorofim (F901318) compared to contemporary antifungal agents against clinical Aspergillus fumigatus isolates, and review of azole resistance phenotype and genotype epidemiology in China. Antimicrob Agents Chemother. 2021;65(5):e02546-20.

166. Rivero-Menendez O, Cuenca-Estrella M, Alastruey-Izquierdo A. In vitro activity of olorofim (F901318) against clinical isolates of cryptic species of Aspergillus by EUCAST and CLSI methodologies. J Antimicrob Chemother. 2019;74(6):1586-90.
167. Buil JB, Rijs A, Meis JF, Birch M, Law D, Melchers WJG, et al. In vitro activity of the novel antifungal compound F901318 against difficult-to-treat Aspergillus isolates. J Antimicrob Chemother. 2017;72(9):2548-52.

168. Badali H, Cañete-Gibas C, Patterson H, Sanders C, Mermella B, Garcia V, et al. In vitro activity of olorofim against clinical isolates of the Fusarium oxysporum and Fusarium solani species complexes. Mycoses. 2021;64(7):748-52.

169. Kennedy T AG, Steiner J, Heep M, Oliver J, Sibley G, Law D., editor Multiple dose pharmacokinetics of an immediate-release tablet formulation of F901318 in healthy male and female subjects. In: Proceedings of the 27th European congress of clinical microbiology and infectious diseases; 22-25 April 2017; Vienna.

170. Kennedy TAG, Steiner J, Heep M, Oliver J, Sibley G, Law D, editor Assessment of the duraiton of infusion on the tolerability and repaet dose pharmacokinetics of F901318 in health-volunteers (abstract P1711). In: Proceedings of the 27th European congress of clinical microbiology and infectious diseases; 22-25 April 2017; Vienna.

171. ClinicalTrials.gov. F901318 single ascending dose study in healthy male volunteers. https://clinicaltrials.gov/ct2/show/ results/NCT02142153?term $=$ F901318\&rslt $=$ With\&draw $=2 \&$ rank=1. Accessed 25 Sep 2021.

172. Tio SH TK, Ng G, Rex JH, Carney D, Slavin M. Olorofim for a case of severe disseminated Lomentospora prolificans infection. In: Proceedings of the 30th European Congress of Clinical Microbiology and Infectious Diseases; 18-21 April 2020; Paris.

173. Chen S RN, Cunneen S, Cornelissen K, Rex JH, Heath CH, Harvey $\mathrm{E}$. A case of Lomentospora prolificans treated with the novel antifungal olorofim. In: Proceedings of the 30th European Congress of Clinical Microbiology and Infectious Diseases; 18-21 April 2020; Paris.

174. Harvey EFL, Rex JH, Thompson G. Successful use of the novel antifungal olorofim in the treatment of dissemianted coccidioidomycosis. Proceedings of the 30th European Congress of Clinical Microbiology and Infectious Diseases; 18-21 April 2020; Paris.

175. ClinicalTrials.gov. Evaluate F901318 treatment of invasive fungal infections in patients lacking treatment options (FORMULA-OLS). https://clinicaltrials.gov/ct2/show/NCT03583164. Acessed 25 Sep 2021.

176. Jenks J, Reed SL, Seidel D, Koehler P, Cornely OA, Mehta SR, et al. Rare mold infections caused by Mucorales, Lomentospora prolificans and Fusarium, San Diego: the role of antifungal combination therapy. Int J Antimicrob Agents. 2018;52(5):706-712.

177. Jenks JD, Seidel D, Cornely OA, Chen S, van Hal S, Kauffman $\mathrm{C}$, et al. Clinical characteristics and outcomes of invasive Lomentospora prolificans infections: analysis of patients in the FungiScope ${ }^{\circledR}$ Registry. Mycoses. 2020;63(5):437-442.

178. Kirchhoff L, Dittmer S, Weisner AK, Buer J, Rath PM, Steinmann J. Antibiofilm activity of antifungal drugs, including the novel drug olorofim, against Lomentospora prolificans. J Antimicrob Chemother. 2020;75(8):2133-40.

179. Cass L, Murray A, Davis A, Woodward K, Albayaty M, Ito K, et al. Safety and nonclinical and clinical pharmacokinetics of PC945, a novel inhaled triazole antifungal agent. Pharmacol Res Perspect. 2021;9(1):e00690.

180. Colley T, Alanio A, Kelly SL, Sehra G, Kizawa Y, Warrilow AGS, et al. In vitro and in vivo antifungal profile of a novel and long-acting inhaled azole, PC945, on Aspergillus fumigatus infection. Antimicrob Agents Chemother. 2017;61(5):e02280-16.

181. Baistrocchi SR, Lee MJ, Lehoux M, Ralph B, Snarr BD, Robitaille $\mathrm{R}$, et al. Posaconazole-loaded leukocytes as a novel treatment strategy targeting invasive pulmonary aspergillosis. J Infect Dis. 2017;215(11):1734-41.

182. Colley T, Sehra G, Daly L, Kimura G, Nakaoki T, Nishimoto Y, et al. Antifungal synergy of a topical triazole, PC945, with a 
systemic triazole against respiratory Aspergillus fumigatus infection. Sci Rep. 2019;9(1):9482.

183. Kimura G, Nakaoki T, Colley T, Rapeport G, Strong P, Ito K, et al. In vivo biomarker analysis of the effects of intranasally dosed PC945, a novel antifungal triazole, on Aspergillus fumigatus infection in immunocompromised mice. Antimicrob Agents Chemother. 2017;61(9):e00124-17.

184. Hoenig1 M. Invasive fungal disease complicating COVID-19: when it rains it pours. Clin Infect Dis. 2020;ciaa1342. https:// doi.org/10.1093/cid/ciaa1342.

185. Thompson GR, Soriano A, Skoutelis A, Vazquez JA, Honore PM, Horcajada JP, et al. Rezafungin versus caspofungin in a phase 2, randomized, double-blind study for the treatment of candidemia and invasive candidiasis: the STRIVE Trial. Clin Infect Dis. 2020; ciaa1380. https://doi.org/10.1093/cid/ciaa1380.

186. Ong V, Hough G, Schlosser M, Bartizal K, Balkovec JM, James $\mathrm{KD}$, et al. Preclinical evaluation of the stability, safety, and efficacy of CD101, a novel echinocandin. Antimicrob Agents Chemother. 2016;60(11):6872-9.

187. Denning DW. Echinocandin antifungal drugs. Lancet. 2003;362(9390):1142-51.

188. Garcia-Effron G. Rezafungin: mechanisms of action, susceptibility and resistance: similarities and differences with the other echinocandins. J Fungi. 2020;6(4):262.

189. James KD, Laudeman CP, Malkar NB, Krishnan R, Polowy K. Structure-activity relationships of a series of echinocandins and the discovery of CD101, a highly stable and soluble echinocandin with distinctive pharmacokinetic properties. Antimicrob Agents Chemother. 2017;61(2):e01541-16.

190. Sandison T, Ong V, Lee J, Thye D. Safety and pharmacokinetics of CD101 IV, a novel echinocandin, in healthy adults. Antimicrob Agents Chemother. 2017;61(2):e01627-16.

191. Ong V, Sandison T, Flanagan S. No relevant pharmacokinetic $(\mathrm{PK})$ interaction between rezafungin and nine probe drugs: results from a drug-drug interaction (DDI) study. Biol Blood Marrow Transplant. 2019;25(3 Suppl.):S357.

192. Pfaller MA, Messer SA, Rhomberg PR, Jones RN, Castanheira M. Activity of a long-acting echinocandin, CD101, determined using CLSI and EUCAST reference methods, against Candida and Aspergillus spp., including echinocandin- and azole-resistant isolates. J Antimicrob Chemother. 2016;71(10):2868-73.

193. Pfaller MA, Messer SA, Rhomberg PR, Castanheira M. CD101, a long-acting echinocandin, and comparator antifungal agents tested against a global collection of invasive fungal isolates in the SENTRY 2015 Antifungal Surveillance Program. Int J Antimicrob Agents. 2017;50(3):352-8.

194. Arendrup MC, Meletiadis J, Zaragoza O, Jørgensen KM, MarcosZambrano LJ, Kanioura L, et al. Multicentre determination of rezafungin (CD101) susceptibility of Candida species by the EUCAST method. Clin Microbiol Infect. 2018;24(11):1200-4.

195. Tóth Z, Forgács L, Locke JB, Kardos G, Nagy F, Kovács R, et al. In vitro activity of rezafungin against common and rare Candida species and Saccharomyces cerevisiae. J Antimicrob Chemother. 2019;74(12):3505-10.

196. Seiler GT, Ostrosky-Zeichner L. Investigational agents for the treatment of resistant yeasts and molds. Curr Fungal Infect Rep. 2021:1-12. https://doi.org/10.1007/s12281-021-00419-5.

197. Helleberg M, Jørgensen KM, Hare RK, Datcu R, Chowdhary A, Arendrup MC. Rezafungin in vitro activity against contemporary Nordic clinical Candida isolates and Candida auris determined by the EUCAST reference method. Antimicrob Agents Chemother. 2020;64(4): $02438-19$.

198. Lepak AJ, Zhao M, VanScoy B, Ambrose PG, Andes DR. Pharmacodynamics of a long-acting echinocandin, CD101, in a neutropenic invasive-candidiasis murine model using an extended-interval dosing design. Antimicrob Agents Chemother. 2018;62(2):e02154-17.

199. Miesel L, Lin KY, Ong V. Rezafungin treatment in mouse models of invasive candidiasis and aspergillosis: Insights on the PK/PD pharmacometrics of rezafungin efficacy. Pharmacol Res Perspect. 2019;7(6):e00546.

200. Wiederhold NP, Locke JB, Daruwala P, Bartizal K. Rezafungin (CD101) demonstrates potent in vitro activity against Aspergillus, including azole-resistant Aspergillus fumigatus isolates and cryptic species. J Antimicrob Chemother. 2018;73(11):3063-7.

201. Wiederhold NP, Najvar LK, Jaramillo R, Olivo M, Wickes BL, Catano G, et al. Extended-interval dosing of rezafungin against azole-resistant Aspergillus fumigatus. Antimicrob Agents Chemother. 2019;63(10):e01165-19.

202. Miesel L, Cushion MT, Ashbaugh A, Lopez SR, Ong V. Efficacy of rezafungin in prophylactic mouse models of invasive candidiasis, aspergillosis, and Pneumocystis pneumonia. Antimicrob Agents Chemother. 2021;65(3):e01992-20.

203. Flanagan S, Goodman DB, Jandourek A, O'Reilly T, Sandison T. Lack of Eefect of rezafungin on QT/QTc interval in healthy subjects. Clin Pharmacol Drug Dev. 2020;9(4):456-65.

204. EMA. Scientific discussion. https://www.ema.europa.eu/en/ documents/scientific-discussion/ecalta-epar-scientific-discu ssion_en.pdf. Accessed 4 Sep 2021.

205. Nyirjesy P, Alessio C, Jandourek A, Lee JD, Sandison T, Sobel JD. CD101 topical compared with oral fluconazole for acute vulvovaginal candidiasis: a randomized controlled trial. J Low Genit Tract Dis. 2019;23(3):22-69.

206. Subcutaneous (SC) injection of CD101, a novel echinocandin: efficacious, well-tolerated and sustained drug exposures 2016. https://n33px2pjph02hfyxt1 xmwn4m-wpengine.netdna-ssl.com/ wp-content/uploads/2015/11/16-ICHS_Ong_SCadmin_PSTR_ FINAL.pdf. Accessed 25 Sep 2021.

207. Soriano AT, GR Vazquez J, Marina-Amadar A, Ilas A, Sanderson T, Pappas PG. Analysis of efficacy from STRIVE phase II trial of rezafungin treatmnet of candaemia and/or invasive candidiasis: outcome during initial day of treatment. 31st ECCMID; 2021; Vienna.

208. Stevens VM, Mueller SW, Reynolds PM, MacLaren R, Kiser TH. Extrapolating antifungal animal data to humans: is it reliable? Curr Fungal Infect Rep. 2020;14(1):50-62.

209. Cidara Therapeutics reports unfavorable results of phase 2 RADIANT trial of CD101 topical in VVC 2017. Available from: http:// ir.cidara.com/node/6481/pdf. Accessed 25 Sep 2021.

210. Locke JB, Almaguer AL, Zuill DE, Bartizal K. Characterization of in vitro resistance development to the novel echinocandin CD101 in Candida species. Antimicrob Agents Chemother. 2016;60(10):6100-7.

211. Cornely OA, Hoenigl M, Lass-Florl C, Chen SC, Kontoyiannis DP, Morrissey CO, et al. Defining breakthrough invasive fungal infection-Position paper of the mycoses study group education and research consortium and the European Confederation of Medical Mycology. Mycoses. 2019;62(9):716-29. 


\section{Authors and Affiliations}

\section{Martin Hoenigl $1^{1,2,3}$ (1) Rosanne Sprute ${ }^{4,5,6} \cdot$ Matthias Egger $^{1} \cdot$ Amir Arastehfar $^{7}$. Oliver A. Cornely $y^{4,5,6,8}$. Robert Krause $^{1}$. Cornelia Lass-Flörl ${ }^{9}$. Juergen Prattes ${ }^{1}$. Andrej Spec ${ }^{10}$. George R. Thompson III $^{11}$. Nathan Wiederhold ${ }^{12}$. Jeffrey D. Jenks ${ }^{2,3,13}$}

1 Division of Infectious Diseases, Department of Internal Medicine, Medical University of Graz, Auenbruggerplatz 15, 8036 Graz, Austria

2 Division of Infectious Diseases and Global Public Health, Department of Medicine, University of California San Diego, La Jolla, San Diego, CA, USA

3 Clinical and Translational Fungal-Working Group, University of California San Diego, La Jolla, San Diego, CA, USA

4 Department I of Internal Medicine, Excellence Center for Medical Mycology (ECMM), University Hospital Cologne, Faculty of Medicine, University of Cologne, Cologne, Germany

5 Chair Translational Research, Cologne Excellence Cluster on Cellular Stress Responses in Aging-Associated Diseases (CECAD), University Hospital Cologne, Faculty of Medicine, University of Cologne, Cologne, Germany

6 German Centre for Infection Research (DZIF), Partner Site Bonn-Cologne, Cologne, Germany

7 Center for Discovery and Innovation, Hackensack Meridian Health, Nutley, NJ, USA
8 Clinical Trials Centre Cologne (ZKS Köln), University Hospital Cologne, Faculty of Medicine, University of Cologne, Cologne, Germany

$9 \quad$ Institute of Hygiene and Medical Microbiology, Medical University of Innsbruck, Innsbruck, Austria

10 Division of Infectious Diseases, Department of Medicine, Washington University School of Medicine, St. Louis, MI, USA

11 Division of Infectious Diseases, Departments of Internal Medicine and Medical Microbiology and Immunology, University of California Davis Medical Center, Sacramento, CA, USA

12 Department of Pathology and Laboratory Medicine, University of Texas Health Science Center at San Antonio, San Antonio, TX, USA

13 Division of General Internal Medicine, Department of Medicine, University of California San Diego, La Jolla, San Diego, CA, USA 